\title{
Numerical Simulation of Shale Gas Flow in Three-Dimensional Fractured Porous Media
}

\author{
Samuel Kazmouz*, Andrea Giusti, Epaminondas Mastorakos \\ Hopkinson Laboratory, Engineering Department, University of Cambridge, UK
}

\begin{abstract}
In this study, a Computational Fluid Dynamics (CFD) solver able to simulate shale gas flow as fluid flow in a porous medium on the macro level is presented. The shale gas flow is described by means of a tailored governing equation with both fluid properties and permeability expressed as a function of the effective pore pressure (stress effect) and with Knudsen effects included through an apparent permeability. This CFD solver, developed in the OpenFoam framework, allows for the simulation of three-dimensional fractured geometries without limitations on the shape of the domain. The solver was assessed and validated against literature data showing good agreement in terms of both recovery rate and pressure field profiles. The solver was then used to explore two different phenomena affecting shale gas dynamics: the diffusion behaviour and the influence of fracture geometry. It was shown that shale gas flow, on the macro level, is a diffusion-dominated phenomenon, and its behaviour can also be qualitatively represented by a diffusion equation. It was also shown that the early behaviour of shale gas flow is dictated by the fracture geometry, and that the reservoir dimensions have no effect on the flow at early times. Finally, a newly developed "dual-zone" solver, where the shale matrix and the fracture network are modelled as two distinct domains interacting through the common boundaries, is presented and discussed.
\end{abstract}

Keywords: Shale gas, Numerical simulation, CFD, OpenFoam, Coupled matrix and fracture zones

${ }^{*}$ Corresponding Author: Samuel Kazmouz Email Address: samuel.kazmouz@lau.edu Tel: +1307 7619078

Preprint submitted to Journal of Unconventional Oil and Gas ResourcesOctober 13, 2016 


\section{Introduction}

In recent years, there has been a renewed interest into alternative hydrocarbon fuels (Youtsos et al. (2013)). Shale gas has become increasingly important after the development of effective technologies for the extraction of these trapped hydrocarbons (Mohaghegh (2013)). In addition to shale gas, shale oil and oil shale constitute part of the current shale hydrocarbon production. It is estimated that the world shale deposits contain around 3 trillion barrels worth of oil (Fan et al. (2010)). Because of this potential for the future energy supply, there is a great interest from the energy industry to improve the understanding of the flow of gas in tight and unconventional reservoirs in order to be able to correctly predict production rates (Ma et al. (2014)).

Several attempts have been done in the past to model the gas flow in shale and tight reservoirs, ranging from analytical and semi-analytical models to numerical simulations. The very early analytical models involved very simple geometries such as a single vertical fracture or a single horizontal fracture (Gringarten et al. (1974)). These early models were followed by semi-analytical models, such as the ones proposed by Patzek et al. (2013) and by Blasingame and Poe (1993). Patzek et al. (2013) studied a very simple configuration of the Barnett shale through a model derived from a non-linear diffusion equation. Desorption was neglected and results were compared with data extracted from real wells giving some insight into the dominant parameters which affect the asymptotic behaviour of the reservoir depletion. Although very fast, most of the analytical and semi-analytical models suffer to capture the non-linearity in shale gas compressibility, viscosity, and compressibility factor due to the use of a pseudo-pressure approach, rather than solving the real gas equation (Houze et al. (2010)). Furthermore, these models also have difficulties in reproducing the typical characteristics of shale gas reservoirs which involve desorption, multiphase flows and complex geometries (Houze et al. (2010)). Recently, some attempts to include non-linearities of shale gas properties in analytical models have been performed (Ma et al. (2014); Wu et al. (2015)), however the applicability to complex reservoirs needs further assessment and there is still need for an approach able to give more detailed information about the shale flow in completely three-dimensional domains.

In this scenario, numerical simulations offer the possibility to capture the non-linearities that in general analytical methods fail to adequately model 
as well as the possibility of accurately reproducing complex reservoir shapes. Furthermore, numerical simulations can be extensively used to perform a sensitivity analysis on the main parameters that affect shale gas production. The main limitations of numerical simulations are related to the computational cost which however is mitigated by the increasing availability of computational resources. Numerical simulations based on a finite element approach were shown to be able to match historic production data of shale gas (Miller et al. (2010); Jayakumar et al. (2011)). Cipolla et al. (2009) investigated some of the parameters which may affect the gas flow, such as the description of the flow from the matrix to the fracture network, stress sensitive fracture conductivity, and desorption. A discrete approach to the grid rather than a dual porosity model approach was utilized and it was concluded that desorption might not be of importance in certain shale reservoirs, but important in others. It was also concluded that the stress effect on the fracture network is more evident during later stages of production rather than at earlier stages and this could lead to optimistic production forecasts (Cipolla et al. (2009)). Further understanding of the shale flow was achieved by Freeman et al. (2013). The major parameters of shale flow were identified as the ultra-tight permeability of shale, configuration of the hydraulically fractured horizontal wells, multiple porosity and permeability fields, and desorption (Freeman et al. (2013)). In addition, three regimes of flow in typical fractured shale reservoirs were noticed: formation linear flow, transitioning into compound formation linear flow, and eventually transforming into elliptical flow (Freeman et al. (2013)). It was also concluded that due to the very low permeability in shale, the flow is controlled by the configuration of the fracture network, with and without desorption effects (Freeman et al. (2013)). Furthermore, Moridis et al. (2010) explored the difference between shale gas reservoirs and tight sand reservoirs using a multiphase solver based on the Darcy equation. It was concluded that these types of reservoirs differ from each other in the contribution of desorption. While desorption can be neglected for tight sand reservoirs, significant deviations from field data are observed if desorption is neglected for shale (Moridis and Freeman (2014)).

Earlier, Kwon et al. (2001) suggested that shale permeability of the Wilcox shale is a function of effective pressure. It was noticed that permeability decreased from $300 \times 10^{-21} \mathrm{~m}^{2}$ to $3 \times 10^{-21} \mathrm{~m}^{2}$ when the effective pore pressure increased from $3 \mathrm{MPa}$ to $12 \mathrm{MPa}$. A cubic power pressure dependent equation of permeability was introduced to best fit the experimental 
values of shale permeability (Kwon et al. (2001)). Later on, Freeman et al. (2011) explored the compositional change of natural gas from shale reservoirs with time. Many reasons were suggested for this phenomenon, but the most important ones are the selective desorption from the surface of the matrix and the non-Darcy flow which is the result of the nano-pores of shale. A dependency between the natural gas composition and the Knudsen number (which controls the non-Darcy flow) and eventually the permeability was suggested. Freeman et al. (2011) placed a large importance on the Knudsen number and used it to alter permeability into an apparent permeability as suggested by Klinkenberg (1941) and Javadpour (2009). Apparent permeability allows retaining the form of the Darcy equation, while capturing the Knudsen effect within the apparent permeability (Freeman et al. (2011)). Further efforts in the understanding and modelling of shale gas flow include a sensitivity analysis of the fracture geometry (Yu et al. (2014)), the use of the finite elements method (Fan et al. (2015)), and a numerical solver that includes slip flow, Knudsen diffusion, and desorption (Shabro et al. (2012)).

Although some aspects of shale gas flow have been already investigated, there is still need of improving the knowledge of shale gas flow in geometries close to the intricate configurations represented by the fracture network of real reservoirs. In order to do that, a solver able to accurately model the shale flow in every kind of geometry is required. In this work a new solver for shale gas flow predictions is proposed and assessed with the main aim of: (i) developing a numerical method able to solve a generic three-dimensional shale reservoir, (ii) analyse the sensitivity of shale gas flow to the shape and the physical properties of the reservoir. The newly developed tools also include a dual domain approach where both the matrix and the fracture are included in the domain and modelled as media with different properties interacting through the common boundaries, offering hence greater accuracy in the flow rate prediction as a function of fracture geometry. Both the mathematical model and the approach used for shale gas simulation make the proposed approach different from the existent commercial solver and models available in literature.

\section{Method}

Shale reservoirs usually consist of a porous material (which in the following will be referred to as matrix) perforated by an intricate network of fractures used to collect the gas trapped in the pores. Despite the porous 
nature of the matrix, the shale gas flow has some peculiarities and cannot be described as the typical flow in porous media.

The major factors affecting shale gas production modelling and eventually forecast are identified as follows. The shale reservoir has a ultra-low permeability and nano-pores, which could lead to a Knudsen diffusion contribution to the flow. This suggests the use of an apparent permeability which includes matrix permeability as well as Knudsen diffusion effects (Javadpour (2009)), while maintaining the use of a Darcy equation. The permeability depends on the effective pressure (stress effect), which is the difference between confining pressure and pore pressure (Kwon et al. (2001)). Due to the ultralow permeability, the fracture network has the largest influence on how the flow proceeds. Finally no consensus has been reached on the role of adsorption. Hill and Nelson (2000) suggest that $20 \%$ to $85 \%$ of total shale storage is in the form of adsorption, however the majority may never be produced. Others suggest that it could be neglected for certain reservoirs (Patzek et al. (2013)). In this work the desorption of shale gas is not considered. This choice is motivated by the fact that, according to the literature (e.g. Patzek et al. (2013)) in the cases used for validation (Barnett shale) the desorption can be neglected. However, it is important to point out that the approach presented here is in principle not limited to cases without desorption since this phenomenon can be included in the formulation through the Langmuir isothermal theory (Shabro et al. (2012)). This will be attempted in future works.

Starting from the typical equations describing the fluid dynamics, a mathematical model for the shale gas flow can be derived (Chen et al. (2006); Gruber (2014)). The following assumptions are considered in the following: (a) single phase flow; (b) gas is assumed to be pure methane (single species); (c) isothermal conditions; (d) negligible gravitational effects; (e) no sources or sinks within the shale matrix; (f) porosity constant in time; (g) permeability is treated as a scalar (isotropic matrix); (h) permeability is a function of effective pressure; (i) no desorption (the gas is only stored within the pore spaces). In the following the mathematical model used in this work is first presented followed by a description of the developed numerical solver and the models adopted for shale properties. All the symbols are defined in Appendix C. 


\subsection{Mathematical model}

The typical representation of a continuum in Computational Fluid Dynamics (CFD) problems, generally involves equations representing the conservation of mass, species, momentum and energy. Since the flow considered here is isothermal and single species (see assumptions (b) and (c)), transport equations for the conservation of energy and specific species are not required (the conservation of methane is expressed through the conservation of mass). In addition, capillary pressures and saturation equations are also not required, because saturation is naturally set to one, with a single phase single species flow.

Following Chen et al. (2006), the conservation of mass can be expressed as:

$$
\frac{\partial \phi \rho}{\partial t}=-\nabla \cdot(\rho u)
$$

where $\phi$ is the porosity, $\rho$ is the density and the source terms on the right hand side have been neglected because of assumption (e). The second equation needed to completely describe the flow within the shale, is the momentum equation. However, since shale is a porous medium, the momentum equation is replaced with the Darcy equation of velocity, which is an empirical equation derived originally for modelling water transport through sand beds. Originally established by Henry Darcy in 1856, this law shows a linear relationship between the fluid velocity and the pressure head gradient (Chen et al. (2006)):

$$
u=-\frac{1}{\mu} k(\nabla P-\rho g \nabla h)
$$

where, $P$ is the pressure, $\mu$ is the fluid viscosity, and $k$ is the permeability. Applying assumption (d) to Eq. 2 yields:

$$
u=-\frac{1}{\mu} k(\nabla P)
$$

In order to close the system, an equation of state is also needed. While in the oil and gas industry cubic equations of state such as the Peng and Robinson (1975) and Soave (1972) equations of state are very common, in this work a real gas law exploiting the Standing and Katz (1942) empirical relationship for the compressibility factor $z$ of natural gas is used (ERCB (1979); Mahmoud (2013)):

$$
\rho=\frac{P W}{z R T}
$$


where, $W$ is the molecular weight, $T$ is the temperature, and $R$ is the methane gas constant. Since the fluid of interest is natural gas, which is a compressible gas, a compressibility $C_{g}$ equation should be introduced:

$$
C_{g}=\left.\left(\frac{1}{\rho} \frac{\partial \rho}{\partial P}\right)\right|_{T}
$$

Combining Eqs. 1, 3 and 4, a material balance for the gaseous species is obtained (Chen et al. (2006)):

$$
\frac{\partial}{\partial t}\left(\frac{\phi P W}{z R T}\right)=-\nabla \cdot\left(\frac{P W}{z R T} \frac{1}{\mu}(-k) \nabla P\right)
$$

Considering assumptions (c) and (f), Eq. 6 can be further simplified into:

$$
\phi \frac{\partial}{\partial t}\left(\frac{P}{z}\right)=\nabla \cdot\left(\frac{P k}{z \mu} \nabla P\right)
$$

Generally (e.g. Patzek et al. (2013)), at this stage of derivation, the compressibility equation is incorporated in Eq. 7, and the pressures are replaced with pseudo pressures (or $P^{2}$ ) to account for the error imposed by assuming that the fluid properties (viscosity, compressibility, porosity, saturation) do not depend on the pressure. However, in this paper, all the fluid properties are taken to be pressure-dependent variables, and hence the need for pseudo pressures is eliminated. As such, this paper takes a different direction, and develops a tailored governing equation. Before further developing Eq. 7, Eq. 5 needs to be manipulated by introducing Eq. 4 and simplifying:

$$
C_{g}=\frac{z R T}{P W}\left(\frac{W}{z R T}-\frac{P W}{z^{2} R T} \frac{d z}{d P}\right)=\frac{1}{P}-\frac{1}{z} \frac{d z}{d P}
$$

Equation 8 can be expressed in the following form by multiplying by $\left(\frac{P \partial P}{z \partial t}\right)$ :

$$
C_{g} \frac{P \partial P}{z \partial t}=\frac{\partial P}{z \partial t}-\frac{P}{z^{2}} \frac{\partial z}{\partial t}
$$

Finally, by replacing the right hand side of Eq. 9 with $\frac{\partial}{\partial t}\left(\frac{P}{z}\right)$, the final form of Eq. 5 is obtained.

$$
\frac{\partial}{\partial t}\left(\frac{P}{z}\right)=\frac{\partial P}{\partial t} \frac{C_{g} P}{z}
$$


Equation 10 is used to manipulate Eq. 7 into the final partial differential equation which describes shale gas flow. Using assumption (f), the right hand side of Eq. 10 can be equated to the right hand side of Eq. 7 multiplied by the porosity $(\phi)$, leading to:

$$
\frac{\partial P}{\partial t} \frac{C_{g} P \phi}{z}=\nabla \cdot\left(\frac{P k}{z \mu} \nabla P\right)
$$

Rearrangement of Eq. 11 yields to the partial differential equation governing shale gas flow:

$$
\frac{\partial P}{\partial t}=\frac{z}{C_{g} P \phi} \nabla \cdot\left(\frac{P k}{z \mu} \nabla P\right)
$$

\subsection{Numerical Methods}

Equation 12 has a similar structure to the diffusion equation (Gruber (2014)) and therefore its implementation into a numerical solver is easier than utilizing a pseudo-pressure equation. A solver for shale gas flow has been implemented in the open source code OpenFOAM (Weller et al. (1998)) where partial differential equations are solved by means of the finite volume approach. The use of the OpenFOAM framework is very useful since it allows for the solution of our model in every kind of geometry, without restrictions. In addition, it allows the use of unstructured grids which facilitate the discretization of complex geometries, typically found in shale gas applications. In order to directly exploit the discretization of the differential operators already available in OpenFOAM, Eq. 12 was rearranged in the following equivalent form which allows an easier implementation:

$$
\frac{\partial P}{\partial t}=\nabla \cdot\left(\frac{k}{\phi \mu C_{g}} \nabla P\right)-\nabla\left(\frac{k}{\phi \mu C_{g}}\right) \cdot \nabla P+\frac{z}{C_{g} P \phi} \nabla\left(\frac{P k}{\mu z}\right) \cdot \nabla P
$$

Once Eq. 13 is solved and the pressure is known, the Darcy velocity can be calculated through Eq. 2 and hence the flow rate can be found. It is important to point out that the reservoir and fluid properties appearing in Eq. 13 can in general be a function of both space and pressure. Permeability, compressibility, compressibility factor, and viscosity, are all treated as spatial variables rather than constants. In addition, these variables will be treated as pressure dependent, as discussed in Section 2.4.

In all the computations performed in this work, the time derivative was discretized using a backward Euler implicit scheme whereas central differ- 
encing second order schemes were used for spatial discretization. If not differently specified, all the computational grids used in this paper are hexahedral meshes (generated using the OpenFOAM meshing tool), but, exploiting the unstructured formulation of the OpenFOAM framework, other meshing strategies can also be used.

Equation 13 can be applied either (i) to the matrix only (single-zone solver) or (ii) to both the matrix and the fracture (dual-zone solver) and both these versions of the solver were implemented. The first case is the simplest scenario where only the matrix needs to be discretized. Boundary conditions are applied to (1) the reservoir confinement, where generally a zero-gradient condition is assumed for the pressure to represent a wall with no flux, but also different types of conditions such as cyclic or constant pressure are possible, and (2) at the interface between the matrix and the fracture, where a constant pressure is usually imposed. In the case of dual-zone solver both the matrix and the fracture should be included in the computational domain and different shale properties should be assigned to these two regions (for example matrix and fracture have different porosity). In the approach followed in this work, the matrix and the fracture were modelled as two different domains interacting through specific boundary conditions at the common interface. A detailed description of the dual-zone solver is given in Section 2.3.

\subsection{Dual Zone Solver}

Petroleum reservoirs usually consists of a low permeability/low porosity matrix and a network of relatively high permeability/high porosity fractures. Historically, the fracture network was natural and, due to the difference in the properties of the matrix and the fracture, this led to the development of dual porosity and dual porosity/dual permeability models in order to better represent the complex geometry of fractures inside a rock matrix (Chen et al. (2006)). While such models were developed long time ago in order to better represent petroleum reservoirs with natural fractures, there seems to be a similar need for shale gas reservoirs, especially with the added complexity of hydraulic fractures.

It would be useful to extend the solver to allow the simulation of the gas flow in both the shale matrix and the fracture network. In order to do that, a dual-zone solver has been implemented where the matrix and the fracture network are treated as two distinct domains, each with its own governing equation and parameters (including permeability and porosity), that only 
interact at their common boundary. This approach is different from the dual porosity model, where the matrix is resembled by blocks and the fracture is resembled by spaces in between the matrix blocks (Chen et al. (2006)), and in principle does not need any transformation or simplification. This offers a great advantage into simulating realistic fracture networks. The dual solver was implemented starting from the dual-zone heat transfer solver developed by Craven and Campbell (2011) for conjugate heat transfer problems.

\subsubsection{Governing Equations}

While the flow within a fracture has been studied and treated as a NavierStokes flow in many studies (Brush and Thomson (2003); Zimmerman and Bodvarsson (1996)), the dual porosity model treats the flow within the fracture as a flow inside a porous medium (Chen et al. (2006)). The fracture is usually characterized by the presence of rock residuals or proppants which in general should be taken into account for an accurate prediction of the flow (Chen et al. (2006)). If Navier-Stokes equations are used, the effect of rock residuals can be included in the simulation only thorough the geometry resulting in a very complex shape of the fracture domain. However, if the flow inside the fracture is treated as a porous medium, the Darcy equation can be used and the effect of rock inside the flow can be taken into account through porosity and permeability values. As a result, in this work the two domains will be treated as porous media with the same governing equation (see Eq. 13). The interaction between these two domains will be controlled through the coupling done at the common boundary. The specific boundary conditions applied to the matrix and the fracture at the common interface are detailed in Sections 2.3.2 and 2.3.3.

\subsubsection{Matrix Boundary Condition}

The boundary condition applied to the matrix at the interface $I$ with the fracture is expressed in terms of pressure. The pressure seen by the matrix should be equal to the pressure on the fracture side. This is a Dirichlet boundary condition and reads as:

$$
\left.P_{m}\right|_{I}=\left.P_{f}\right|_{I}
$$

\subsubsection{Fracture Boundary Condition}

Inspired by the dual porosity model, which states that the flow rate of the gas leaving the matrix enters the fracture network, the boundary condition 
for the fracture, at the fracture-matrix interface, is a Neumann boundary condition. The introduction of the mass flow rate leaving the matrix into the fracture as a boundary condition is done through the pressure gradient. By imposing that the mass flow rate leaving the matrix is equal to the flow entering the fracture and after introducing the Darcy expression (Eq. 2) for the velocity and removing the area which is a common factor, the following expression for the boundary condition at the common interface $I$ is obtained:

290

$$
\begin{aligned}
\left.\dot{m}_{f}\right|_{I}=\left.\dot{m}_{m}\right|_{I} \rightarrow \rho_{f} A u_{f, n}=\rho_{m} A u_{m, n} & \\
& \left.\rightarrow \rho_{f} \frac{k_{f}}{\mu_{f}} \nabla P_{f, n}\right|_{I}=\left.\rho_{m} \frac{k_{m}}{\mu_{m}} \nabla P_{m, n}\right|_{I}
\end{aligned}
$$

$$
\left.\nabla P_{f, n}\right|_{I}=\left.\frac{\rho_{m}}{\rho_{f}} \frac{k_{m}}{k_{f}} \frac{\mu_{m}}{\mu_{f}} \nabla P_{m, n}\right|_{I}
$$

Hence the coupling is done by imposing on the fracture side the component of the pressure gradient normal to the interface according to Eq. 16. If this coupling algorithm is working properly within the solver, considering also that at the interface the matrix and the fracture have the same pressure (see Eq. 14) and therefore the same density, the normal component of the Darcy velocity at the common boundary should be be equal for each zone. This will be used later on for validation.

\subsection{Natural Gas and Shale Properties}

In order to properly describe the evolution of shale gas, physical properties appearing in Eq. 13 need to be accurately modelled. In the following, the main modelling assumptions for the physical properties of shale gas (methane is considered here) are summarized together with some considerations regarding the porosity and permeability of the matrix. It is important to point out that the code is not limited to the use of the following relations. In principle, every kind of relation for the physical properties can be implemented and used making the approach very flexible and ready to incorporate a more comprehensive description of the properties or to be extended to different fuels and applications.

\subsubsection{Compressibility factor}

Methane has a critical temperature of $190 \mathrm{~K}$ and a critical pressure of $4600 \mathrm{kPa}$ (Friend et al. (1989)). The pressure and temperature of the natural gas found within shale formations exceeds the critical values. Hence, 
methane will not behave as an ideal gas within shale. Originally, Standing and Katz (ERCB (1979)) developed plots of the compressibility factor for sweet natural gas versus reduced pressure and temperature. These charts were curve-fit by Dranchuk, Purvis, and Robinson, using the BWR equation of state (ERCB (1979)). However, Mahmoud (2013) showed that the original equations are not accurate at high pressures, and suggested another set of equations which better matches compressibility factor values at high pressure.

$$
\begin{gathered}
z=a P_{r}^{2}+b P_{r}+c \\
a=0.702 e^{-2.5 T_{r}} \\
b=-5.524 e^{-2.5 T_{r}} \\
c=0.044 T_{r}^{2}-0.164 T_{r}+1.15
\end{gathered}
$$

where the reduced pressure and the reduced temperature are given by:

$$
P_{r}=\frac{P}{P_{c r}}, \quad T_{r}=\frac{T}{T_{c r}}
$$

Furthermore, Jarrahian et al. proposed a cubic equation of state which allows the calculation of the compressibility factor of natural gas with higher accuracy (Jarrahian and Heidaryan (2014)), and can be seen in Appendix B. In this code, due to the flexibility of the OpenFOAM framework, any adequate equation of state can be implemented, depending on the composition of the gas and the properties of the reservoir.

As a result, compressibility factor was implemented into the code as a pressure dependent property using both, Eq. 17 (Mahmoud (2013)) and Eq. B.1 (Jarrahian and Heidaryan (2014)) (Eq. B.1 is the virial form of the cubic equation of state presented in Jarrahian and Heidaryan (2014)). The results shown in Section 3 were produced using Eq. 17, while the results produced using Eq B.1 are included in Appendix A.

\subsubsection{Compressibility}

Similar to the compressibility factor, compressibility has an empirical equation which was developed by Dranchuk, Purvis, and Robinson (ERCB 
(1979)). However, since compressibility can be calculated from the equation of compressibility factor (Eq. 17), we will use the relation presented by Mahmoud (2013), which provides better accuracy at high pressures. The compressibility is described by the following set of equations (Mahmoud (2013)):

$$
\begin{gathered}
C_{g}=\frac{C_{r}}{P_{r}} \\
C_{r}=\frac{1}{P_{r}}-\frac{1}{z}\left(\frac{\partial z}{\partial \rho_{r}}\right)_{T_{r}} \\
C_{r}=\frac{1}{P_{r}}-\frac{1}{z}\left[P_{r}\left(1.404 e^{-2.5 T_{r}}\right)-\left(5.524 e^{-2.5 T_{r}}\right)\right]
\end{gathered}
$$

where the reduced pressure $P_{r}$ and reduced temperature $T_{r}$ were defined in Eq. 18. In Fig. 1, the compressibility and compressibility factor predicted using Eqs. 17, B.1 and 19 are compared with the equations suggested by Dranchuk, Purvis, and Robinson (ERCB (1979)). The three methods give very similar values for a reduced pressure less than 1.0 whereas for higher pressures (pressure larger than the critical value) the effect of the corrections suggested by Jarrahian and Heidaryan (2014) and by Mahmoud (2013) is clearly visible.

\subsubsection{Viscosity}

The viscosity of natural gas, and in this case pure methane, varies by a factor of four between pore pressure and fracture pressure (Ling (2010)). Hence, for the sake of mathematical rigour as well as exploiting the advantage of numerical simulation, viscosity will also be pressure dependent. The Lee-Gonzalez-Eaken correlation (Gonzalez et al. (1970)) was developed by measuring the viscosity of eight natural gases over a range of temperatures and pressures. This correlation can be seen in Eq. 20 (Ling (2010)). 


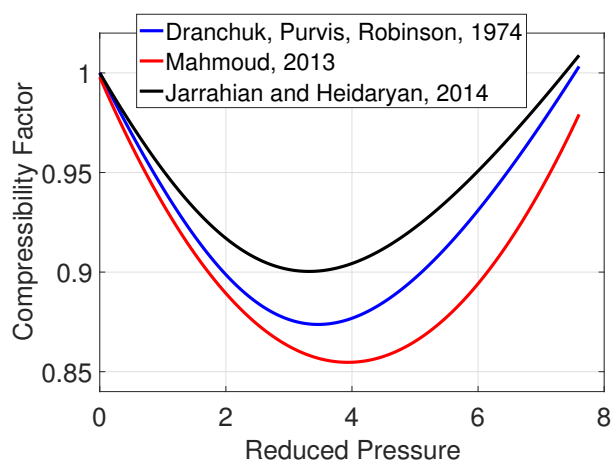

(a) Compressibility Factor.

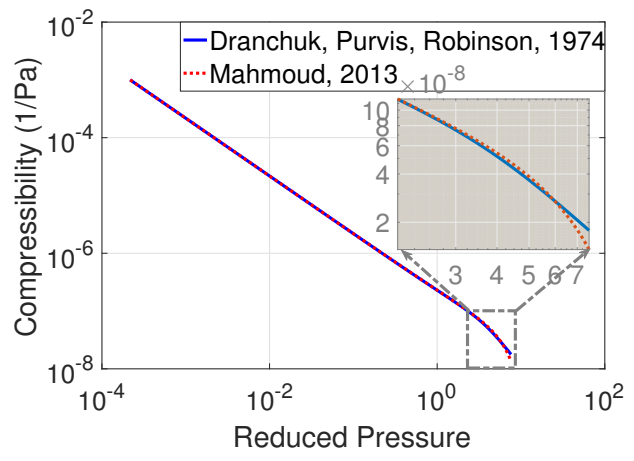

(b) Compressibility.

Figure 1: Compressibility factor and compressibility versus reduced pressure, produced using the equations from Refs. ERCB (1979), Jarrahian and Heidaryan (2014), and Mahmoud (2013).

$$
\begin{gathered}
\mu=10^{-4} K \exp \left(X \rho^{Y}\right) \\
K=\frac{(9.379+0.01607 W) T^{1.5}}{209.2+19.26 W+T} \\
X=3.448+\left[\frac{986.4}{T}\right]+0.01009 W \\
Y=2.447-0.2224 X \\
\rho=\frac{P W}{z R T}=0.00149406 \frac{P W}{z T}
\end{gathered}
$$

where $\mu$ is in cp (centipoise), $P$ in psia, $\rho$ in $\mathrm{g} / \mathrm{cm}^{3}$, and $T$ in ${ }^{\circ} \mathrm{R}$.

In addition, Jarrahian et al. (2015) suggested a different viscosity correlation, based on their own cubic equation of state, which provides better accuracy at higher pressures and temperatures for sour and sweet natural gases. This correlation can be seen in Eq. B.2 (Jarrahian et al. (2015)), and can be found in Appendix B. The values of viscosity predicted by Eq. 20 and Eq. B.2 are shown in Fig. 2. The results shown in Section 3 were produced using Eq. 20, while the results produced using Eq B.2 are included in Appendix A. 


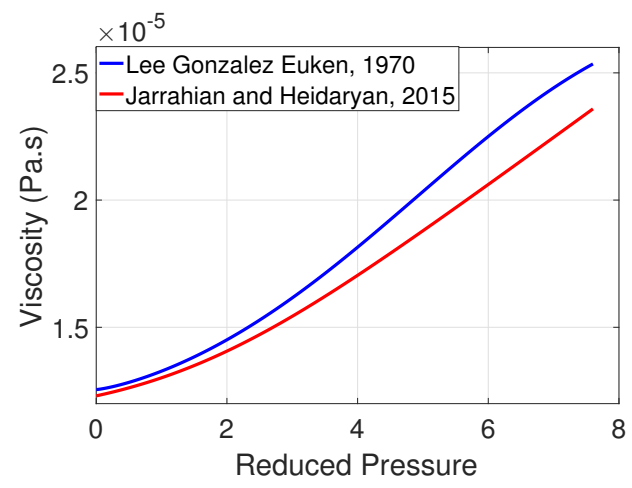

Figure 2: Viscosity versus reduced pressure using Eq. 20 andEq. B.2

\subsubsection{Permeability}

In general, reservoirs with permeability below $10^{-15} \mathrm{~m}^{2}(1.013 \mathrm{mD})$ are considered tight (Wang et al. (2014)). This dictates that shale, which is classified as a tight reservoir, will have a permeability lower than $10^{-15} \mathrm{~m}^{2}(1.013$ $\mathrm{mD})$. So Soeder (1988) reported a shale permeability of $2 \times 10^{-17} \mathrm{~m}^{2}(20.26$ $\mu \mathrm{D})$. Bruner et al. (2011) reported values within the range of $2 \times 10^{-17} \mathrm{~m}^{2}$ $(20.26 \mu \mathrm{D})$ to $1 \times 10^{-16} \mathrm{~m}^{2}(100.13 \mu \mathrm{D})$. In 2006 , the US geological survey published a report which compiled shale sample results from several shale formations. This report states that shales with liquid petroleum in their pores had very low permeabilities in the range of tens of nano-darcies $\left(1 \times 10^{-20} \mathrm{~m}^{2}\right)$ (Milici and Swezey (2006)). With many shale reservoirs containing oil and gas, permeabilities in the range of nano-darcies remain realistic. Keeping in mind that hydraulic fractures are created to increase permeability and that naturally occurring fractures passively increase permeability, one cannot standardize a constant permeability for shale. Even further, some works suggest that permeability depends on pressure. For example, the permeability of illite-rich shale of the Wilcox formation has been found to decrease from $3 \times 10^{-19} \mathrm{~m}^{2}(304 \mathrm{nD})$ to $3 \times 10^{-21} \mathrm{~m}^{2}(3.04 \mathrm{nD})$ when the effective pressure increases from $3 \mathrm{MPa}$ to $12 \mathrm{MPa}$ (Kwon et al. (2001)). As a result, this paper utilizes a permeability equation which depends on effective pressure (Kwon et al. (2001)):

$$
k=10^{-17}\left[1-\left(\frac{P_{e}}{19.3 \times 10^{6}}\right)^{0.159}\right]^{3}
$$


The effective pressure, $P_{e}$, is the difference between confining pressure, the pressure due to the weight of the rock on top of the reservoir, and the pore pressure. Confining pressure $P_{c}$ is estimated at $38 \mathrm{MPa}$ throughout the paper.

In addition, as noted in Section 1, it was suggested that the Darcy equation for velocity is not sufficient to represent shale gas flow, which is a slip-flow or a transition flow. However, Javadpour (2009) showed that we can indeed maintain the Darcy form, but we need to use a specific form of permeability, called apparent permeability, which takes into account both the effect of the physical geometry of the rock (Darcy permeability) and the flow (slip and Knudsen effects). Apparent permeability is also used in this paper according to the following expression (Javadpour (2009)):

$k_{a}=\frac{2 r \mu W}{3 \times 10^{3} R T \rho_{\text {avg }}^{2}}\left(\frac{8 R T}{\pi W}\right)^{0.5}+\left(\frac{r^{2}}{8 \rho_{\text {avg }}}\right)\left[1+\left(\frac{8 \pi R T}{W}\right)^{0.5} \frac{\mu}{p_{\text {avg }} r}\left(\frac{2}{\alpha}-1\right)\right]$

In Eq. 22, $\alpha$ is called the tangential momentum accommodation coefficient (the fraction of the gas molecules reflected diffusely), and ranges between 0 and 1.

The values of permeability given by Eqs. 21 and 22 as a function of pressure are shown in Fig. 3 (pore radius was assumed to be $2 \mathrm{~nm}$, and $\alpha$ was assumed to be 0.5). In Fig. 3(a), permeability increases as pressure increases. Physically, this can be attributed to the aperture of the pores. As the pore pressure increases, it resists against the confining pressure and keeps the physical pore volume large, which enhances the flow and subsequently increases permeability (elastic deformation is minimal). On the other hand, as the pore pressure decreases, the confining pressure starts to crush the pores, decreasing their volume, and subsequently reduce permeability (elastic deformation is significant) (Kwon et al. (2001)). In addition, when natural or hydraulic fractures exist, the same effect can be noticed, which leads to the use of proppant to maintain an adequate fracture aperture and to enhance recovery. Nevertheless, the use of proppants does not infinitely improve recovery, as the fractures and the proppant will eventually be crushed, and this gives shale gas its characteristic sharp production drop which is usually mitigated by drilling many wells. On the contrary, Fig. 3(b) shows that the apparent permeability decreases as pressure increases. Permeability has a value slightly smaller than $1 \times 10^{-19} \mathrm{~m}^{2}(101 \mathrm{nD})$ for pressure equal to $35 \mathrm{MPa}$ (this is the initial reservoir pressure throughout this paper), which is the Darcy permeability. As discussed in (Javadpour (2009)), for pressures 


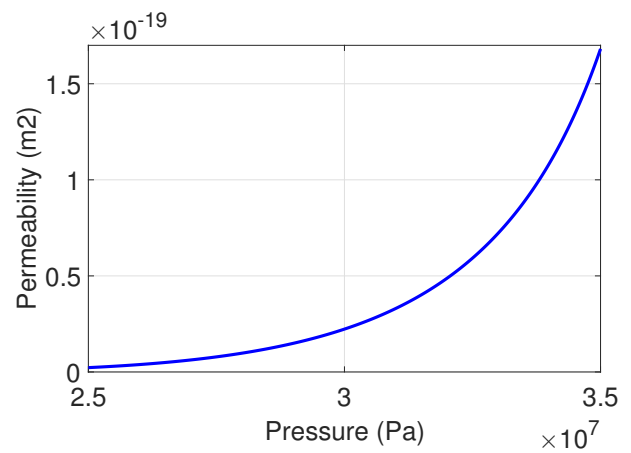

(a) Pressure-Dependent Perm. (Eq. 21)

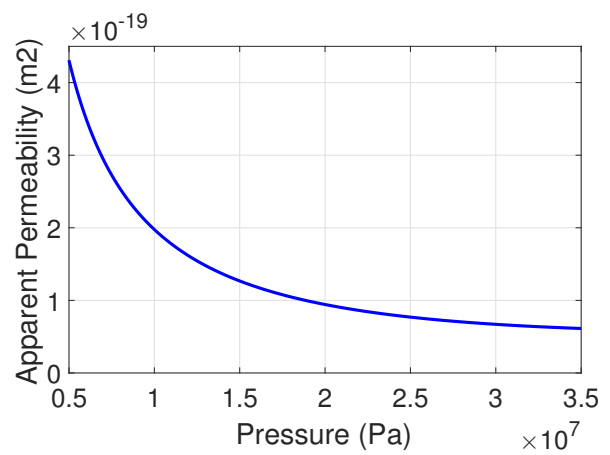

(b) Apparent Perm. (Eq. 22)

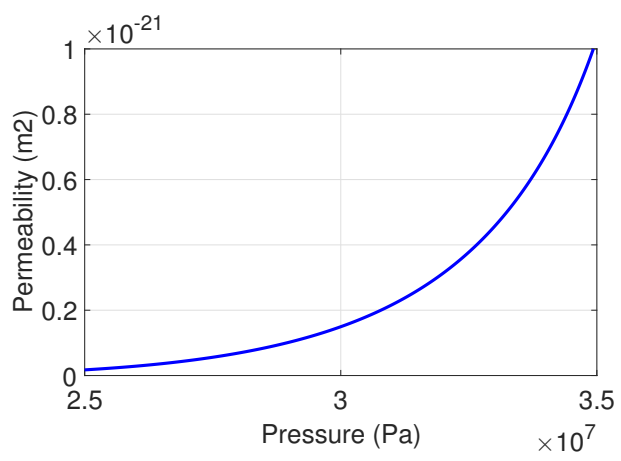

(c) Pressure-Dependent and Apparent

Perm. (Eq. 21 and Eq. 22 combined)

Figure 3: Permeability versus pressure using Eqs. 21 and 22, from Refs. Kwon et al. (2001); Javadpour (2009).

larger than $10 \mathrm{MPa}$, the Darcy and the apparent permeability have a ratio of 1.0. As pressure decreases, the ratio of apparent to Darcy permeability increases significantly because the mean free path increases, and the flow shifts into slip and Knudsen regimes. The apparent to Darcy permeability ratio at the pressure of $5 \mathrm{MPa}$ is around 5 , as can be deduced from Fig. 3(b) by comparing the permeability values at $35 \mathrm{MPa}$ and $5 \mathrm{MPa}$. This observation is consistent with results presented by Javadpour (2009). Finally, the permeability resulting from the combined effects of pressure dependence and apparent permeability is shown in Fig. 3(c). In order to combine both Eq. 21 and Eq. 22, the constant $10^{-17}$ in Eq. 21 was replaced by the permeability calculated from Eq. 22. The term $10^{-17}$ can be seen as a base permeability being adjusted by the pressure (stress effect). The permeability is equal to 
the base value for $P_{e}=0$ and decreases as the effective pressure increases. This effect is also replicated in Fig. 3(c), where the use of Eq. 22 for the base value results in lower values of the permeability. This should be considered an attempt to include both the stress and Knudsen effects in the same formulation.

\subsubsection{Porosity}

Although porosity is not a homogeneous and constant property, it is simpler to assume it as a constant, especially when the shale is assumed to be isotropic (assumptions (f) and (g)). It was estimated that the average porosity of the Barnett and Marcellus shales is 6\% (Bruner et al. (2011)). Other sources suggest a range of porosity between $8.2 \%$ and $11.4 \%$ for the Marcellus shale (Taylor (2013)). As such, a value of $9 \%$ porosity will be used in this study. Note, however, that our governing equation (Eq. 13) allows for $\phi$ variations in space, if needed.

\section{Results and Discussion}

First, an assessment and validation of the model proposed here will be presented followed by the analysis of the diffusion behaviour and the influence of the fracture geometry. An example of simulation of a very complex fracture geometry is also included. Both the validation and the analysis of the behaviour of shale gas flow were performed using the single-zone solver, where only the matrix was included in the computational domain. The generic case solved here includes a horizontal well, with two vertical orthogonal pennyshaped fractures, as shown in Fig. 4. Finally, an application of the dual-zone solver, showing the potentiality of this approach, is presented and discussed.

\subsection{Validation}

The model is validated against two sets of literature results. The first one is the work of Patzek et al. (2013), where a two-regime flow is described. The second one is the work of Freeman et al. (2013), where the shale gas flow exhibits three types of flow: formation linear flow, compound linear flow, and elliptical flow.

\subsubsection{Two-Regime Flow}

In the work of Patzek et al. (2013) thousands of well data, from the Barnett, were analysed and compared with a one-dimensional model for shale 

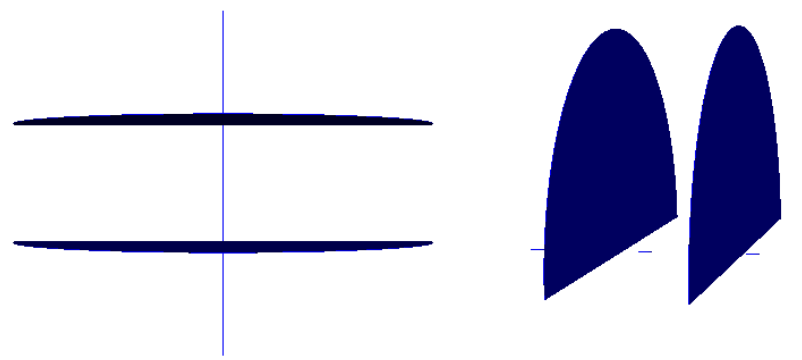

Figure 4: Generic fracture geometry.

gas production. It was shown that the fractional cumulative gas production (Recovery Factor, RF) before fracture interference is proportional to a factor $\kappa$ multiplied by the square root of a normalized dimensionless (scaled) time $\tilde{t}$ :

$$
\begin{gathered}
\operatorname{RF}(\tilde{t}) \approx \kappa \sqrt{\tilde{t}} \\
\tilde{t} \equiv t / \tau \quad, \quad \tau=d^{2} / \alpha_{i} \\
\alpha_{i}=\left.\frac{k}{\phi S_{g} \mu_{g} C_{g}}\right|_{\text {Initial reservoir } P, T}, \quad R F(\tilde{t})=m / M
\end{gathered}
$$

where $\tau$ is the time elapsed before the occurrence of fracture interference, which depends on the initial conditions of the reservoir as well as the distance between two adjacent fractures but not on the reservoir dimensions. It was also shown that the recovery rate (or mass flow rate) exhibits a tworegime flow. In the first regime, the recovery rate declines at a rate inversely proportional to the square root of the dimensionless (scaled) time. The second regime is an exponential decline which occurs after fracture interference.

The one-dimensional model proposed by Patzek et al. (2013) is based on the same governing equations described in this study, but with a different treatment of the pressure dependence of the shale properties for which a pseudo-pressure notation was introduced. On the other hand, the formulation proposed in this work solves for the flow in three dimensions, retaining the governing equation in absolute pressure form (without resorting to pseudo pressures because all the fluid properties are implemented as functions 


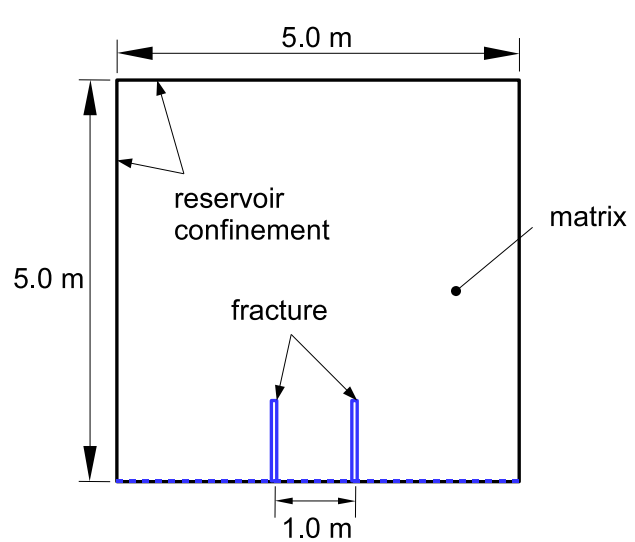

(a) Case 1.

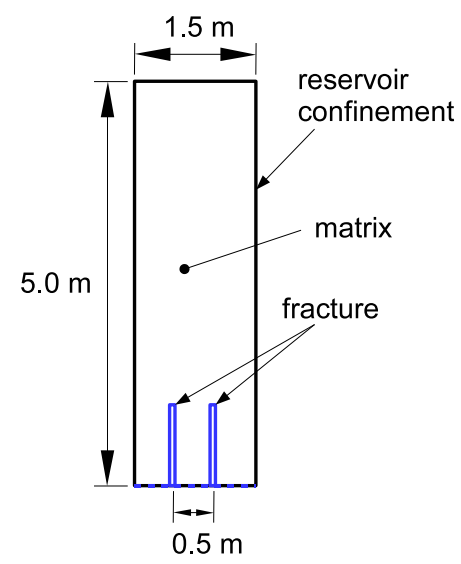

(b) Case 2 .

Figure 5: Schematic of the two cases investigated for the assessment of the two-regime flow.

of pressure). Since in the model derived in this work an isotropic matrix is assumed, a behaviour similar to the one found by Patzek et al. (2013) is also expected from our computations. Therefore, the capability of the present approach to capture the two-regime flow will be evaluated in the following as a first step of validation.

Two different cases were solved here as schematically shown in Fig. 5. Both cases use the generic fracture geometry shown in Fig. 4 which is equivalent to the uniformly spaced hydrofracture stages in a horizontal well considered in Patzek et al. (2013), and differ for the spacing between the fracture elements and the dimension of the reservoir. The relevant properties of each case are shown in Table 1. A uniform pressure equal to 5.0 MPa was imposed at the fracture boundary whereas the zero-gradient condition (no outflow) was applied at the reservoir walls. An initial pore pressure of $35.0 \mathrm{MPa}$ was applied in both cases.

While Patzek et al. (2013) assumed a constant permeability, deduced from Eq. 23 through a direct comparison with experimental measurements (for the investigated wells, a value in the range of $5 \times 10^{-19} \mathrm{~m}^{2}(506 \mathrm{nD})$ to $5 \times 10^{-20} \mathrm{~m}^{2}$ (50.6 nD)was found), here different permeability models (PM) were considered and applied to each case, therefore assessing the effect of permeability on the shale flow prediction. Table 2 summarizes the different permeability models used here. First, a constant permeability of $1 \times 10^{-19} \mathrm{~m}^{2}(101 \mathrm{nD})$ 
Table 1: Properties of the two generic cases solved.

\begin{tabular}{lll}
\hline \hline Case & Case 1 & Case 2 \\
\hline Total Length & $5 \mathrm{~m}$ & $5 \mathrm{~m}$ \\
Total Width & $5 \mathrm{~m}$ & $1.5 \mathrm{~m}$ \\
Total Height & $5 \mathrm{~m}$ & $5 \mathrm{~m}$ \\
Fracture Half Length & $0.9 \mathrm{~m}$ & $0.9 \mathrm{~m}$ \\
Fracture Spacing & $1.0 \mathrm{~m}$ & $0.5 \mathrm{~m}$ \\
Fracture Thickness & $3 \mathrm{~mm}$ & $3 \mathrm{~mm}$ \\
Porosity & $9 \%$ & $9 \%$ \\
$\alpha$ & 0.5 & 0.5 \\
Pore Radius & $2 \mathrm{~nm}$ & $2 \mathrm{~nm}$ \\
Temperature & $330 \mathrm{~K}$ & $330 \mathrm{~K}$ \\
Critical Pressure & $4.6 \mathrm{MPa}$ & $4.6 \mathrm{MPa}$ \\
Critical Temperature & $190 \mathrm{~K}$ & $190 \mathrm{~K}$ \\
Molar Mass $\left(M_{w}\right)$ & $16.04 \mathrm{~kg} / \mathrm{kmol}$ & $16.04 \mathrm{~kg} / \mathrm{kmol}$ \\
Number of Elements & $1.6 \mathrm{million}$ elements & $0.9 \mathrm{million}$ elements \\
Time Step & $60 \mathrm{~seconds}$ & $60 \mathrm{~seconds}$ \\
Initial Pore Pressure & $35 \mathrm{MPa}$ ( $)$ & $35 \mathrm{MPa}$ \\
Outlet (Fracture) BC & Dirichlet (5 MPa or 25 MPa) & Dirichlet (5 MPa or 25 MPa) \\
Reservoir BC & Neumann (Zero-Gradient) & Neumann (Zero-Gradient) \\
Characteristic Time $\tau$ & 8.44 hours or 844 hours & 2.11 hours or 211 hours \\
\hline \hline
\end{tabular}

was assumed (PM1); second, the dependence on the effective pressure (stress effects, see Eq. 21 Kwon et al. (2001)) was accounted for (PM2); third, the apparent permeability (Knudsen effects, see Eq. 22 (Javadpour (2009))) was considered (PM3); finally, by combining Eq. 21 and Eq. 22, both the stress and Knudsen effects were included (PM4). An average pore radius $r=2 \mathrm{~nm}$ and tangential momentum accommodation coefficient $\alpha=0.5$ were assumed, when using apparent permeability.

It is important to note that in reality, actual permeability values are larger than the values experimentally tested in labs, due to the effect of hydraulic fracturing on permeability values in the vicinity of the fracture/matrix interface (Patzek et al. (2013)). Equation 21 from Kwon et al. (2001), is a lab-based relationship and because of that will provide permeability values lower than actual wells. Therefore, it is expected that when this expression is used, a lower recovery rate will be produced. This relation was tested for an effective pressure range of $3 \mathrm{MPa}$ to $12 \mathrm{MPa}$ (Kwon et al. (2001)), and as such, the boundary condition at the fracture surface was increased to $25 \mathrm{MPa}$ for PM2 and PM4, to maintain the effective pressure within the tested range. Figure 6 shows a 3D cut of the computational mesh used for Case 1. 
Table 2: Summary of the four permeability models (PM) used in this work.

\begin{tabular}{ll}
\hline \hline Permeability Model (PM) & Main Feature \\
\hline Permeability Model 1 (PM1) & Constant Permeability $\left(1 \times 10^{-19} \mathrm{~m}^{2}\right)(101 \mathrm{nD})$ \\
Permeability Model 2 (PM2) & Stress Effect (Effective Pressure Permeability, Eq. 21 Kwon et al. (2001)) \\
Permeability Model 3 (PM3) & Knudsen Effect(Apparent Permeability, Eq. 22 Javadpour (2009)) \\
Permeability Model 4 (PM4) & Stress Effect and Knudsen Effect (Eq. 21 and Eq. 22) \\
\hline \hline
\end{tabular}

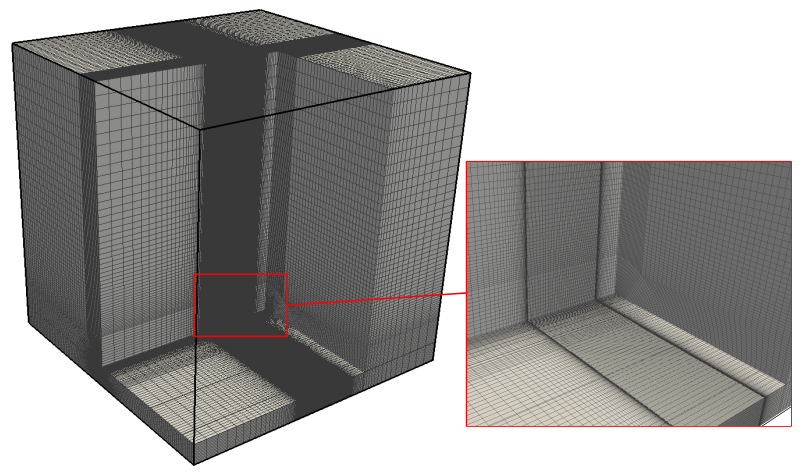

Figure 6: Computational mesh used for Case 1.

Refinements around the fracture were adopted in order to properly solve the pressure gradients, usually very steep in this region. A similar grid was also used for Case 2 and for all the other cases investigated in this work, except the complex fracture geometry presented in Section 3.4 for which a tetrahedral mesh was used. An example of time evolution of the pressure and permeability (PM3) inside the matrix is shown in Fig. 7 where snapshots of the solution at different times are reported. As time advances, the pressure around the fracture drops and hence the permeability increases, which matches the trend in Fig. 3-b (Since PM3 is utilized in this case). Although in Section 3, the solution is analysed mainly in terms of global quantities (such as recovery rate and recovery factor), the outcome of the solver is the three-dimensional pressure field which allows us to monitor the evolution of the shale quantities in any region of the domain.

Since the fracture spacing in Case 1 is double the one in Case $2(1.0 \mathrm{~m}$ and $0.5 \mathrm{~m}$, respectively), the characteristic time $\tau$ for Case 1 is four times the characteristic time for Case 2 because the fracture spacing in Eq. 23 is squared. With the initial pore pressure of $35 \mathrm{MPa}$ and initial reservoir temperature of $330 \mathrm{~K}$ (Table 1), the two cases investigated here have a characteristic interference time $\tau$ of 8.44 and 2.11 hours respectively, for PM1-PM3. 


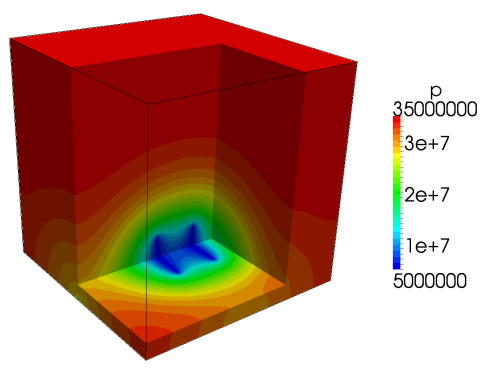

(a) Pressure $(t=172 h)$

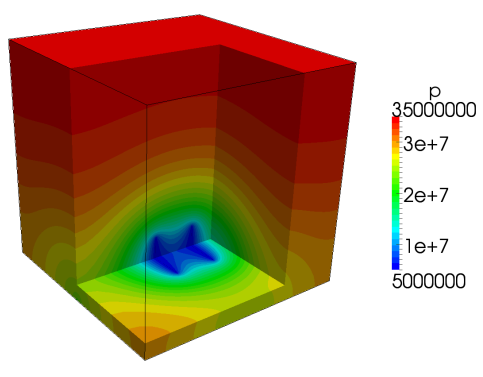

(c) Pressure $(t=344 h)$

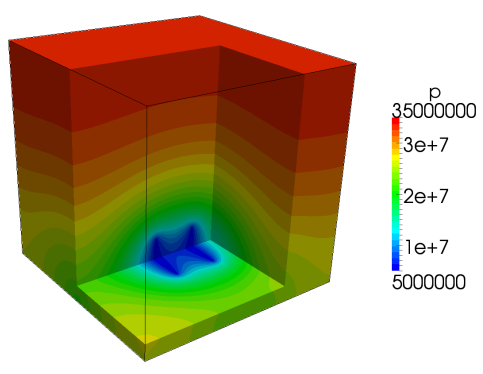

(e) Pressure $(t=516 h)$

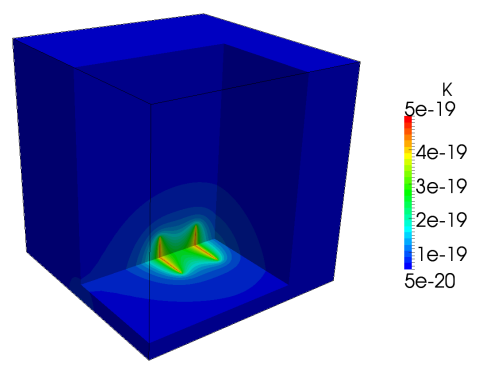

(b) Permeability $(\mathrm{t}=172 \mathrm{~h})$

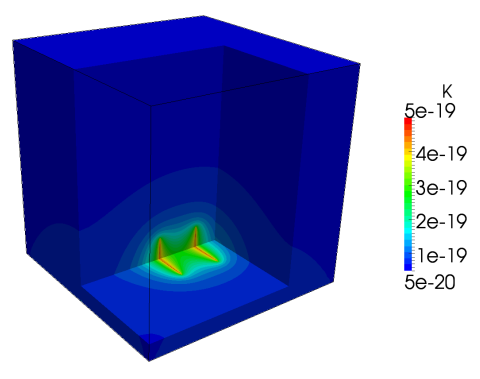

(d) Permeability $(\mathrm{t}=344 \mathrm{~h})$

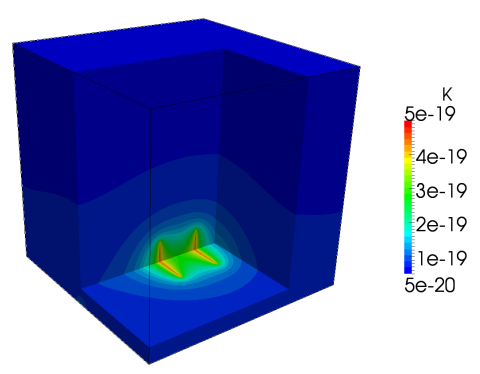

(f) Permeability $(t=516 h)$

Figure 7: Pressure and permeability plots at several times, for Case 1 using PM3

530

However, for PM4, when permeability at the initial conditions is $1 \times 10^{-21} \mathrm{~m}^{2}$ $(1.01 \mathrm{nD})$, see Fig. 3(c), interference time $\tau$ increases to 844 and 211 hours respectively.

Figures 8 and 10 show the recovery factor and the recovery rate versus the scaled time, obtained for Case 1 using the different permeability models. 


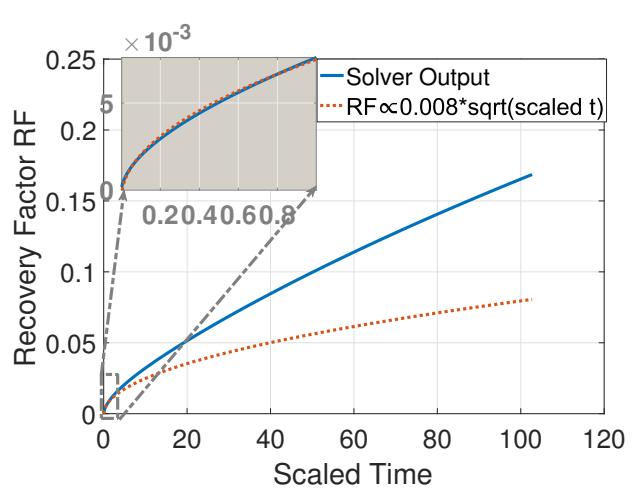

(a) PM1.

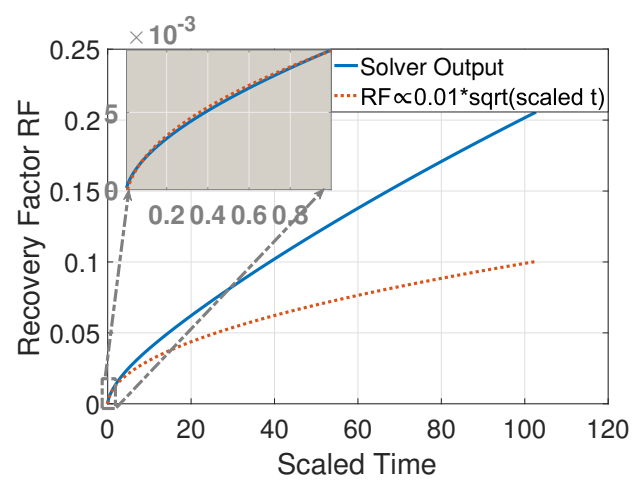

(c) PM3.

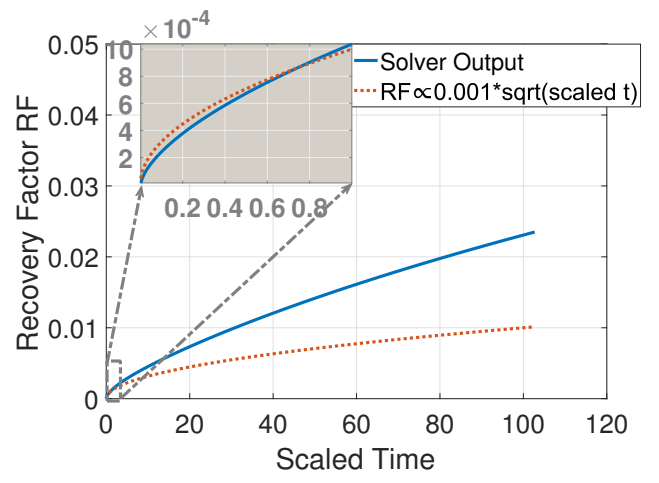

(b) PM2.

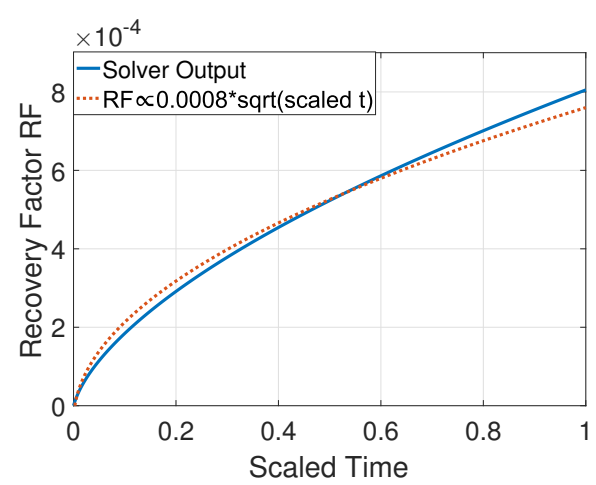

(d) PM4.

Figure 8: Recovery factor versus scaled time for Case 1 predicted using the four permeability models (PM), with curve matching.

Similarly, results for Case 2 are reported in Figs. 9 and 11. All the results show an agreement with the two-regime flow previously described and for both Case 1 and Case 2, the use of apparent permeability (PM3) allows for a better match of the profile inversely proportional to the square root of the scaled time observed by Patzek et al. (2013) before fracture interference. Apparent permeability is the key feature that distinguishes the shale gas flow from other porous material, and in general should be included in the formulation. It is also interesting to note that the recovery rate and recovery factor are very sensitive to the value of the permeability and different predictions are obtained with the various models. Therefore, great attention should be devoted to the selection of the right model for permeability.

It is also interesting to look at the value of the constant $\kappa$ appearing in 


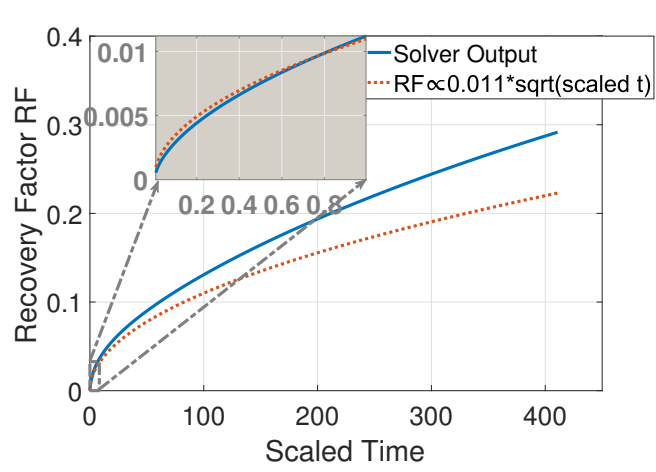

(a) PM1.

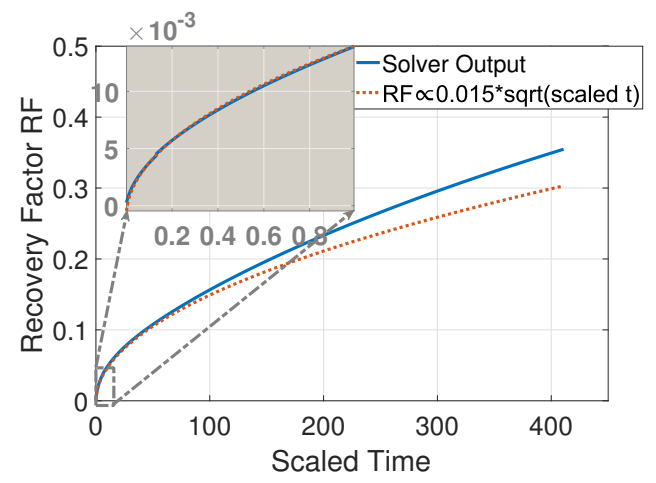

(c) PM3.

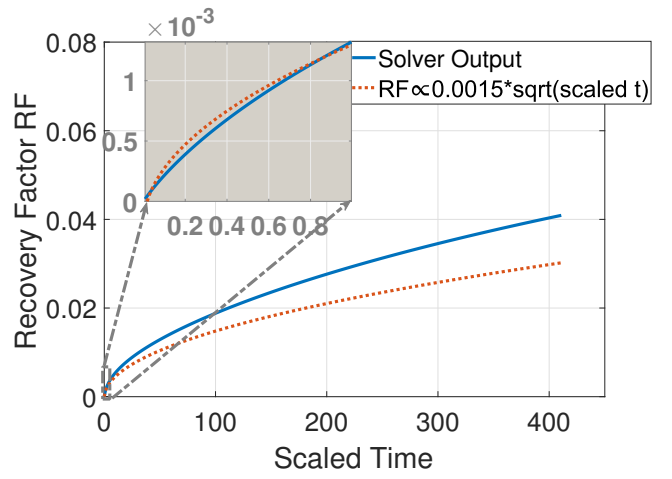

(b) PM2.

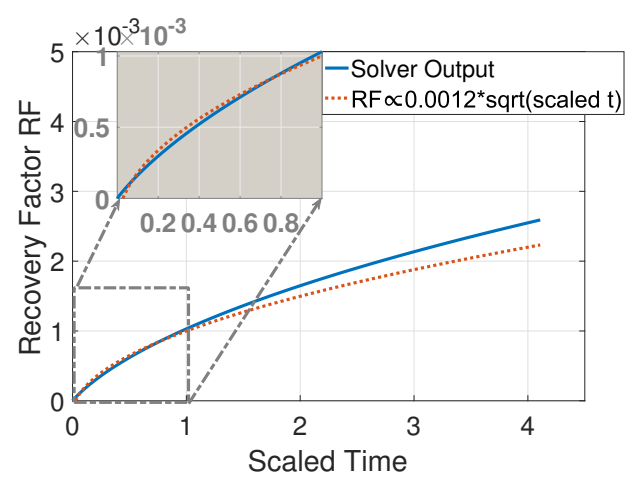

(d) PM4.

Figure 9: Recovery factor versus scaled time for Case 2 predicted using the four permeability models (PM), with curve matching.

the expression of the recovery factor vs. scaled time during the early stages of the reservoir depletion (Eq. 23). This constant depends on the fracture geometry, the permeability of the shale, the gas properties, as well as the reservoir size. For the Barnet shale wells, a value around 0.625 was found by Patzek et al. (2013) whereas for the geometries investigated in this work a value in the range 0.001-0.015 seems to give a good scaling. Assuming that in real application the fracture network is created through hydraulic fracturing, for optimal (fastest) recovery, $\kappa=0.625$ can be regarded as a practical (rather than theoretical) upper limit. The fracture networks used in this paper are far from being optimized for recovery. Hence, $\kappa$ is far lower than the value suggested by Patzek et al. (2013). However, when comparing the two cases (see Fig. 8 and Fig. 9), it should be noted that Case 2 depletes 


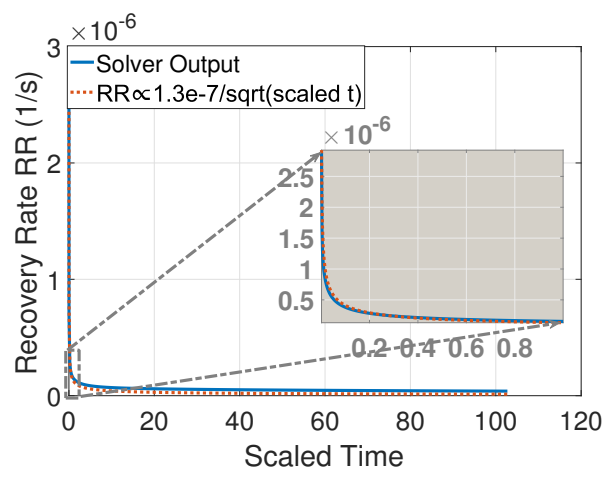

(a) PM1.

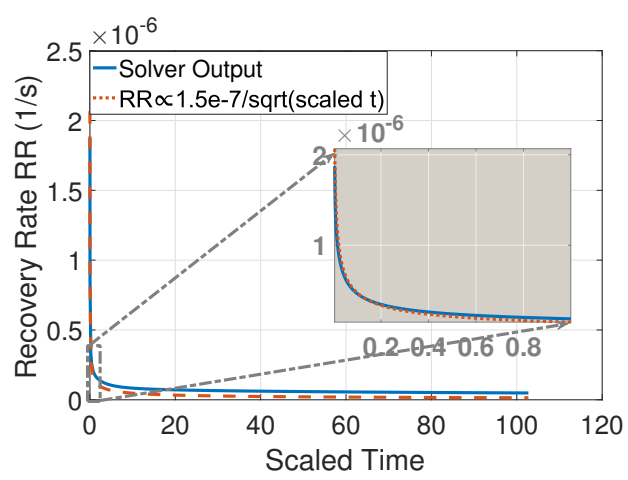

(c) PM3.

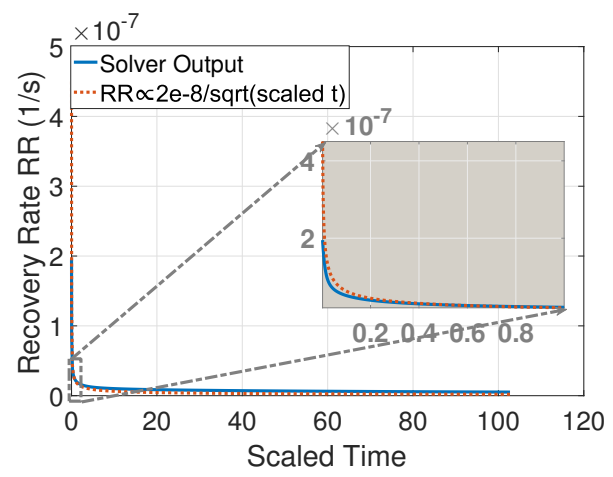

(b) PM2.

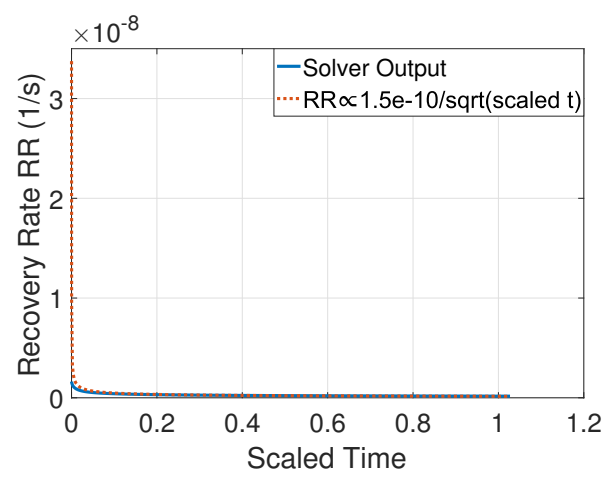

(d) PM4.

Figure 10: Recovery rate versus scaled time for Case 1 predicted using the four permeability models (PM), with curve matching.

much faster than Case 1. This can be attributed to the reservoir size and to the characteristic interference time $\tau$. For the same fracture network, a larger reservoir reduces the constant $\kappa$, because of the larger amount of natural gas in the reservoir, which naturally requires a longer time to extract. In addition, a larger characteristic interference time $\tau$ reduces the constant $\kappa$, because it takes a longer time for fracture interference to occur.

The results shown in this section demonstrate that the three-dimensional shale gas flow still preserves the two-regime flow characteristics described by Patzek et al. (2013), regardless of the model used to include the permeability. However, the recovery rate is in general very sensitive to the permeability, and therefore great care should be used for the selection of the more consistent permeability model, being both stress and Knudsen effects 


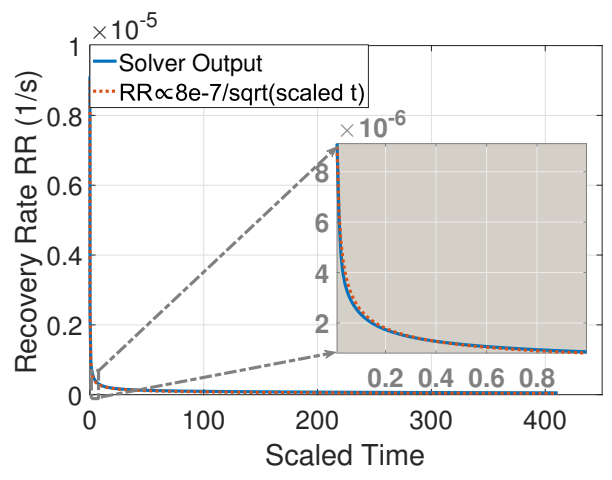

(a) PM1.

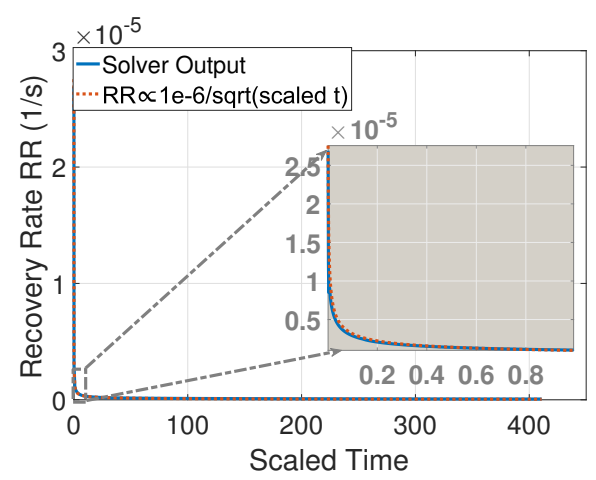

(c) PM3.

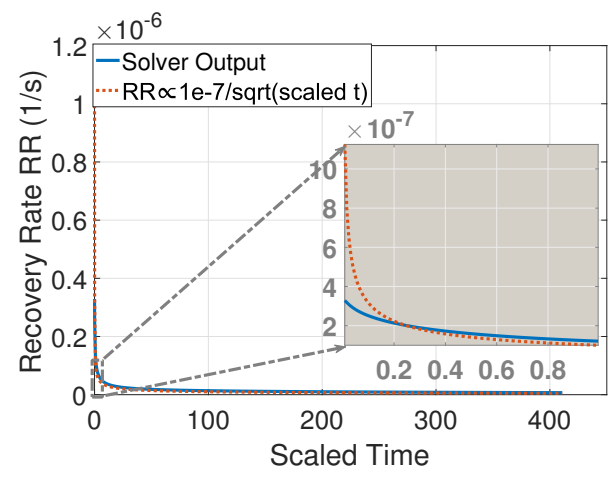

(b) PM2.

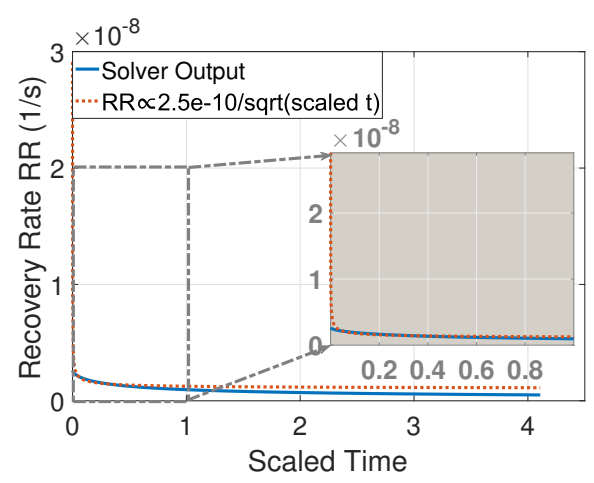

(d) PM4.

Figure 11: Recovery rate versus scaled time for Case 2 predicted using the four permeability models (PM), with curve matching.

in general non-negligible.

\subsubsection{Three-Regime Pressure Contours}

In the work of Freeman et al. (2013), the pressure field inside the matrix was directly analysed and on the basis of the different shapes of the pressure iso-lines. Three flow regimes were distinguished: formation linear flow, compound formation linear flow, and elliptical flow. In order to qualitatively validate the solver presented in this study, the pressure contours obtained in the previous simulations are compared to those presented by Freeman et al. (2013). Although the dimensions of the fracture and the well network used here are different compared to the geometry used in (Freeman et al. (2013)), the trends of the pressure profiles can still be compared. Figure 12 shows 

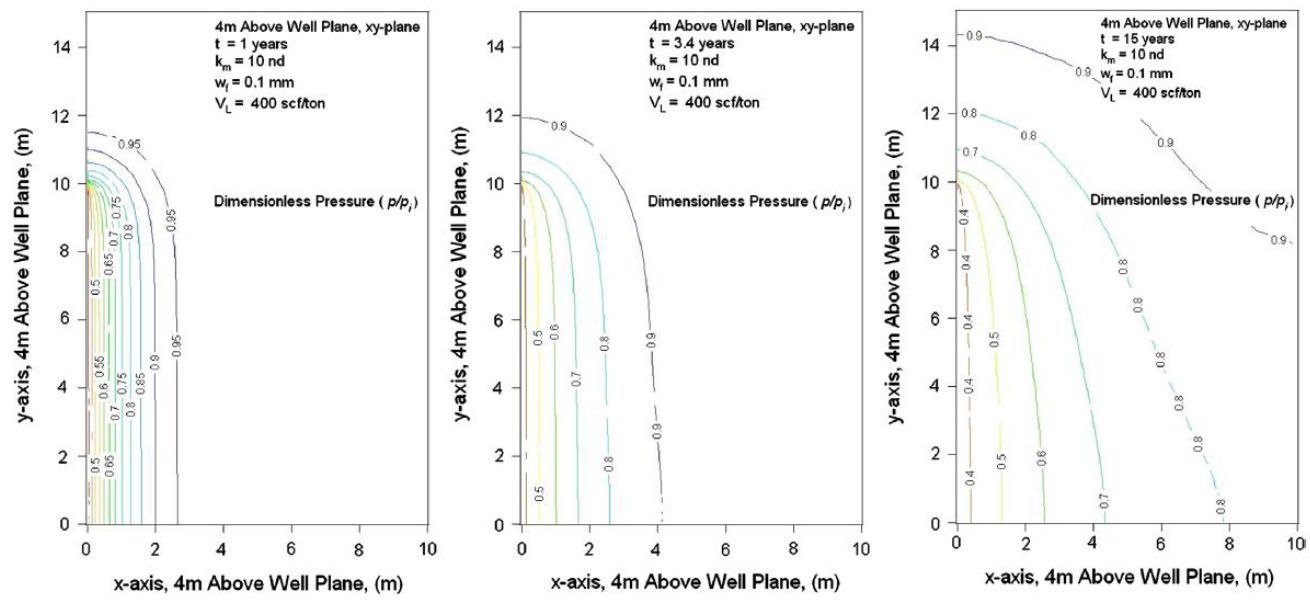

Figure 12: Left: formation linear flow, Center: compound linear flow, Right: elliptical flow Freeman et al. (2013). (Reused with persmission from Elsevier. License Number: $3902540332270)$

the three flow regimes around the fracture from the work by Freeman et al. (2013). The linear flow seen in Fig. 12 is characterized by parallel pressure iso-lines. As the flow proceeds into compound linear and elliptical flow, the iso-lines spread around the fracture and are not parallel anymore but transform into elliptical profiles.

Fig. 13 shows the pressure profiles from the solution of Case 2 (using a variable permeability function). Qualitatively, the same transition into the three regimes of Fig. 12 was found. While the geometric dimensions are different between the two studies, the comparison demonstrate the capability of the present approach to capture the required physics, showing agreement with literature pressure regimes around the fracture.

Furthermore, Freeman et al. (2013) suggest that the transition between formation linear flow and compound linear flow is triggered by fracture interference which causes a change of regime in the recovery rate. The formation linear flow and the compound linear flow are determined by the fracture geometry and fracture interference. Fig. 13 shows that the transition between the formation linear flow and the compound linear flow happens at around a time equal to $\tau$ (i.e. scaled time $=1$ ). According to Freeman et al. (2013), this change in flow regime will be matched by a change in recovery rate regime. Fig. 14 locates this regime change in a plot showing the recovery 


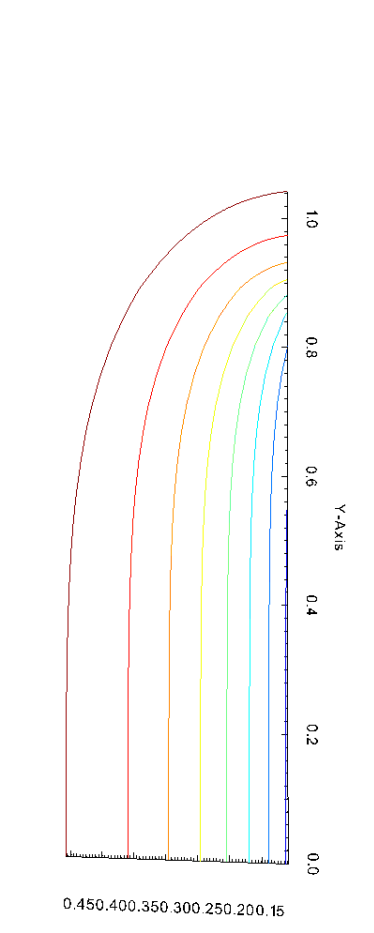

Scaled Time $=0.6$

Formation Linear Flow
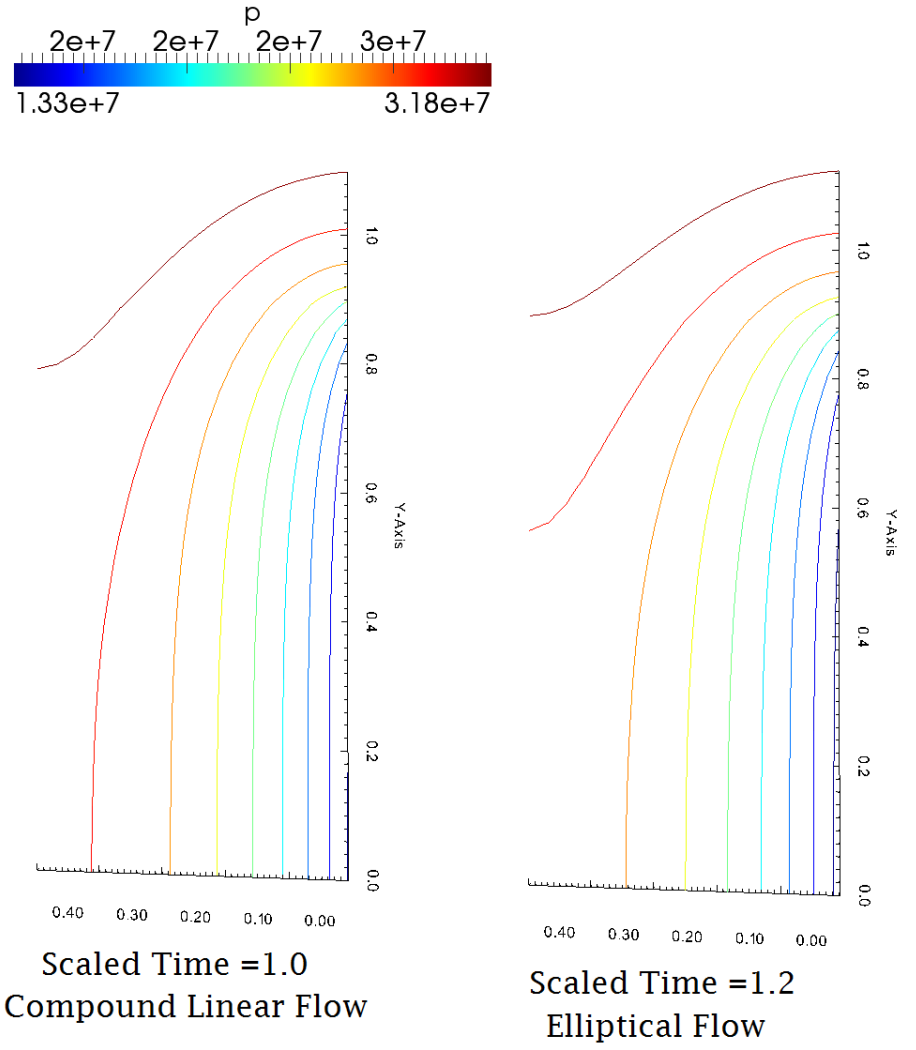

Figure 13: Left: formation linear flow, Centre: compound linear flow, Right: elliptical flow

rate vs. scaled time. This can be linked back to the work of Patzek et al. (2013) and the two-regime flow analysed in Section 3.1.1. For $\tau<1$, where the recovery factor is proportional to the square root of scaled time, the pressure contour does not show fracture interference. Therefore the square root regime of the recovery factor can be associated with the absence of interference between the fractures (i.e. formation linear flow in the pressure contours). The transition from formation linear flow to compound linear flow around the fracture triggers the regime change in recovery rate at a scaled time of 1.0. After fracture interference, the profile of the flow is expected to be affected by the global geometry of the fracture network and the recovery rate turns into exponential decay (Patzek et al. (2013)) if the fracture network is optimized for recovery, or a different profile depending on the global characteristics of the fracture. In this second stage, the interaction with the 


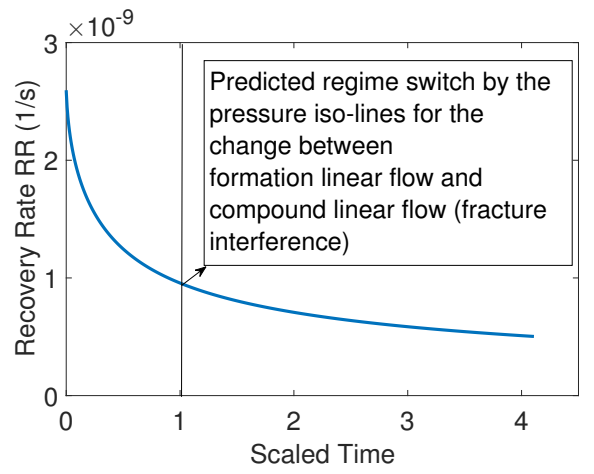

Figure 14: Recovery rate versus time, with the regime change highlighted at scaled $t=1$

reservoir confinement may also play an important role on the evolution of the shale flow. This will be further investigated in Section 3.3.

\subsection{Diffusion Behaviour}

Although shale gas flow is often described as a diffusion phenomenon (Patzek et al. (2013)), the governing equation (see Eq. 13) is not a pure diffusion equation because of the two extra terms appearing on the right hand side. In order to investigate the contribution of these two terms to the shale flow, results obtained in Section 3.1 for Case 1 (see Table 1) will be compared with results from a reduced model, where only the diffusion term in Eq. 13 is retained (for this test, PM4 was utilized):

$$
\frac{\partial P}{\partial t}=\nabla \cdot\left(\frac{k}{\phi \mu C_{g}} \nabla P\right)
$$

This equation is quite simple to implement and solve and can be viewed as a simplified model of the shale gas flow.

Comparisons between the solution obtained with the complete model (Eq. 13, already discussed in Section 3.1) and the diffusion-only model (Eq. 24) are shown in Fig. 15 where the logarithmic plot of the recovery factor and the recovery rate $v s$. time are reported. It is possible to note that the use of Eq. 24 leads to an overestimation of the recovery rate, and therefore an overestimation of the recovery factor, compared to the complete model. This means that the second and third terms on the right hand side of Eq. 13 have the effect of reducing the diffusion mass flow rate.

Considering the depletion time, the difference between the two cases is significant and this suggests that Eq. 24 cannot be used to model shale gas 


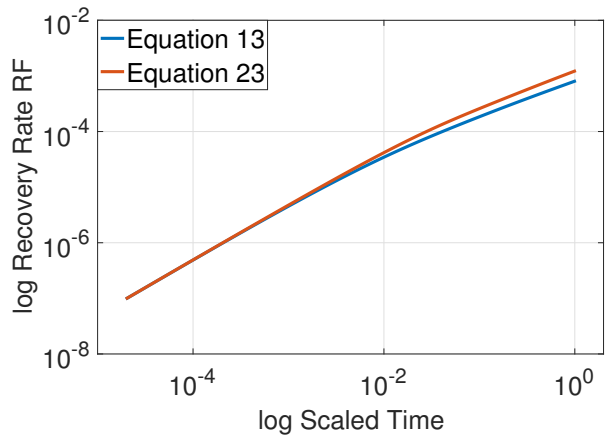

(a) Logarithmic plot of the recovery factor vs. time.

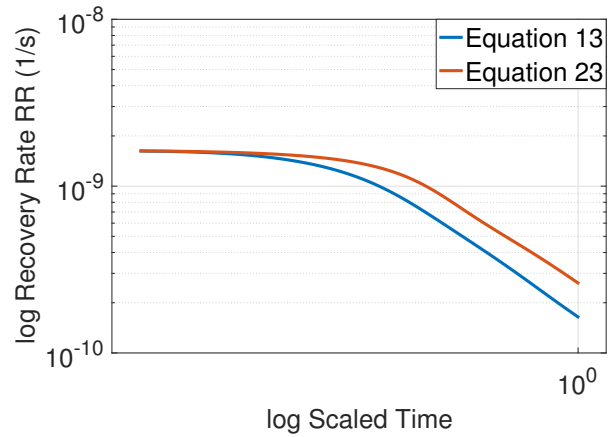

(b) Logarithmic plot of the recovery rate $v s$. time.

Figure 15: Comparison between the shale gas behaviour predicted by the complete model (Eq. 13) and the diffusion-only model (Eq. 24).

when accuracy is a critical factor. However, the trends in Fig. 15 show that the solution of Eq. 24 still preserves the two-regime flow. Therefore, although the additional terms in the complete model have an important impact on the mass flow rate, the physical behaviour seems dominated by diffusion.

The results in this section show that the flow of natural gas in shale is indeed a diffusion-dominated phenomenon. A diffusion equation can properly model the two-regime behaviour, but gives very different predictions of quantities relevant for practical purposes, such as the recovery factor and the recovery rate, compared to the complete model.

\subsection{Influence of the geometry}

Natural gas flow in shale is known to be strongly influenced by the fracture network geometry (Patzek et al. (2013)). In order to further investigate this aspect, four geometries with the same fracture network but with varying reservoir sizes were solved. In all the cases the reservoir has the shape of a parallelepiped with the same area of the cross section but different lengths, as schematically shown in Fig. 16 and detailed in Table 3. Simulations with variations of the other dimensions of the reservoir (for example the width) were also performed (refer to Appendix A), leading to the same conclusions.

The results in Fig. 17 show that the recovery rate and the recovery factor are the same for all geometries. Therefore it is possible to conclude that the flow behaviour is determined by the fracture network, regardless of the dimension of the domain. Furthermore, Fig. 17 shows that all four geometries 


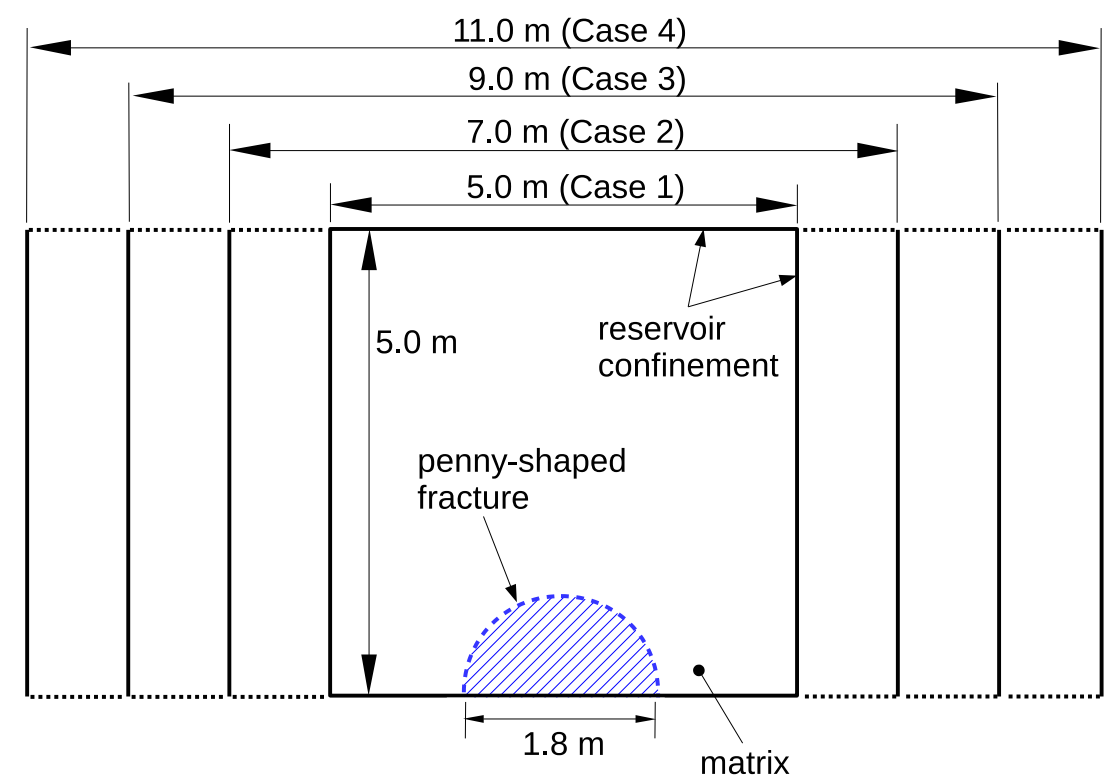

Figure 16: Schematic of the cases considered for the sensitivity analysis to the reservoir dimension.

Table 3: Geometrical properties of the geometries used to investigate the effect of fracture network

\begin{tabular}{llll}
\hline Geometry & Reservoir Length & Reservoir Height & Reservoir Width \\
\hline Geometry 1 & $5 \mathrm{~m}$ & $5 \mathrm{~m}$ & $5 \mathrm{~m}$ \\
Geometry 2 & $7 \mathrm{~m}$ & $5 \mathrm{~m}$ & $5 \mathrm{~m}$ \\
Geometry 3 & $9 \mathrm{~m}$ & $5 \mathrm{~m}$ & $5 \mathrm{~m}$ \\
Geometry 4 & $11 \mathrm{~m}$ & $5 \mathrm{~m}$ & $5 \mathrm{~m}$ \\
\hline \hline
\end{tabular}

produce the same amount of gas after a given time is elapsed. This means that, regardless of size, early shale gas behaviour is set by the fracture network. Only in the last stages of the depletion, when the flow interacts with the boundary walls, the reservoir shape and dimension can affect the shale production. In other words, the recovery rate is mainly dependent on the fracture geometry, until boundary conditions of the reservoir come into effect. This further validates the solver, as this behaviour is expected and agreed upon in literature, as already discussed in Patzek et al. (2013); Freeman et al. (2013). 


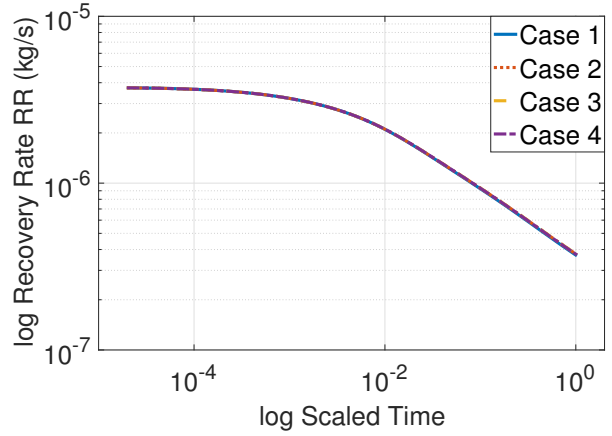

(a) Logarithmic plot of recovery rate vs. scaled time

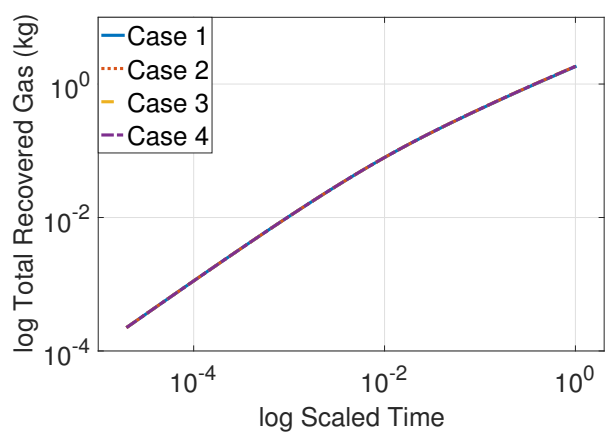

(b) Logarithmic plot of recovery vs. scaled time

Figure 17: Sensitivity analysis to the dimension of the reservoir.

\subsection{Complex Geometry}

As stated in Section 1, one of the main properties of shale gas reservoirs, is the complexity of the fracture geometry. Since one of the main aims of this work is to present a three-dimensional CFD solver able to solve very complex fracture networks, it would be adequate to show the capability of the solver to predict the shale flow in a more realistic fracture geometry. The geometry was confined inside a block of matrix whose dimensions are $0.5 \times 0.5 \times 0.1 \mathrm{~m}^{3}$, and can be seen in Fig. 18. The permeability model PM3 (see Table 2) was used to generate the results. Due to the complex geometry, in this case the domain was discretized by means of a tetrahedral mesh generated using the software ICEM, part of the ANSYS package. A time sequence of the pressure and permeability distributions in the domain, is shown in Fig. 19.

It is interesting to note how in complex fracture geometries, the interaction between the different fractures happens at different times being controlled by the inter-distance between the various fracture elements. As shown in Fig. 19, in the region where two fracture elements are very close to each other the pressure is quite low (higher local depletion) whereas where the distance between the elements is bigger, the higher level of pressure indicates that a larger amount of shale gas is still present in that region. The three-dimensional solver presented here is able to capture all these features and solving in detail the shale flow around the fracture and, in principle, the solution can also be used to calibrate low-order analytical models. For the sake of completeness, the global recovery factor and the recovery rate are reported in Fig. 20. The maximum recovery factor achieved in this case 


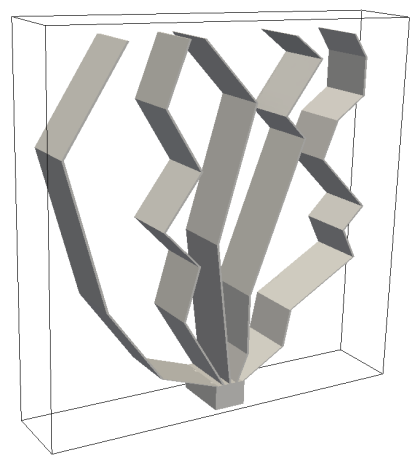

Figure 18: Fracture geometry of the case presented in Section. 3.4

(see Fig. 20(b)) is around 62\%. This is due to the outlet boundary condition set to $5 \mathrm{MPa}$, well above atmospheric pressure, which does not allow the complete depletion of the reservoir.

\subsection{Dual Zone Solver}

In principle, the mathematical model describing the shale behaviour can be used to study the shale flow in both the matrix and the fracture. Keeping in mind that the shale matrix and the fracture network vary greatly in permeability and porosity, the domain can be decomposed into two sub-domains, corresponding to the matrix and the fracture respectively, where different properties are assigned. This is the principle behind the dual zone solver implemented in this work (see Section 2.3) where the governing equation of the single zone solver is applied to both the matrix and the fracture and the interaction between the two regions is imposed through specific coupling conditions at the common interface. In order to distinguish between the two regions, specific values of permeability and porosity should be assigned, consistent with the nature of the matrix and the fracture. With the main aim of showing the capability of this approach, the dual zone solver has been used to investigate the simple test case shown in Fig. 21. Although the solver can be applied to any kind of geometry, as the ones used for the single zone solver, this case was chosen for the very simple shape of the interface which facilitates the meshing of the two domains (a one-to-one correspondence of the faces at the interface is required in the current implementation) and at the same time reduces the computational cost. The zone parameters used in 


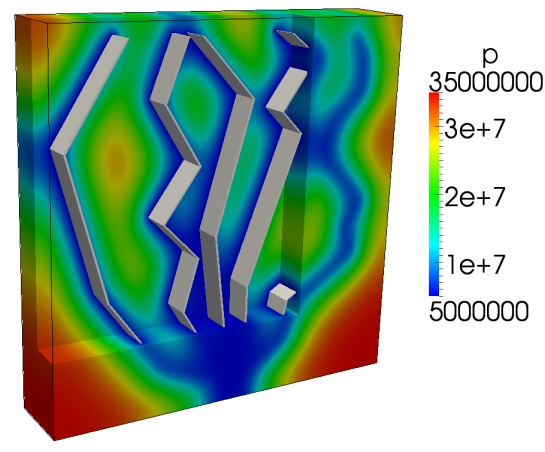

(a) Pressure, Time $=0.18 \mathrm{~h}$

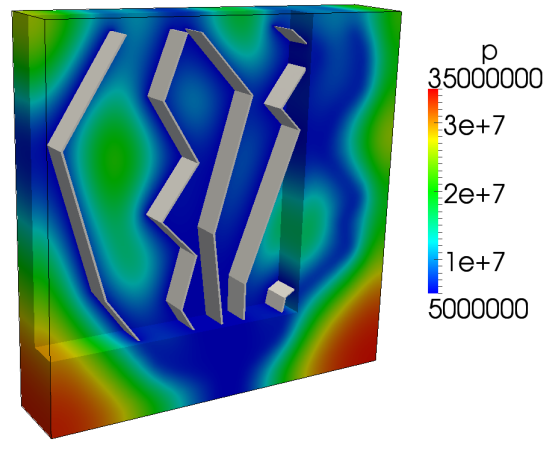

(c) Pressure, Time $=0.36 \mathrm{~h}$

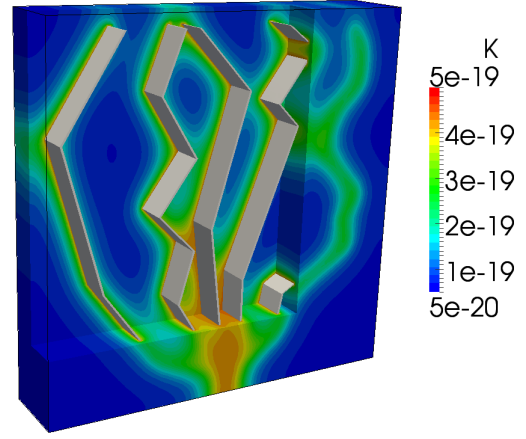

(b) Permeability, Time $=0.18 \mathrm{~h}$

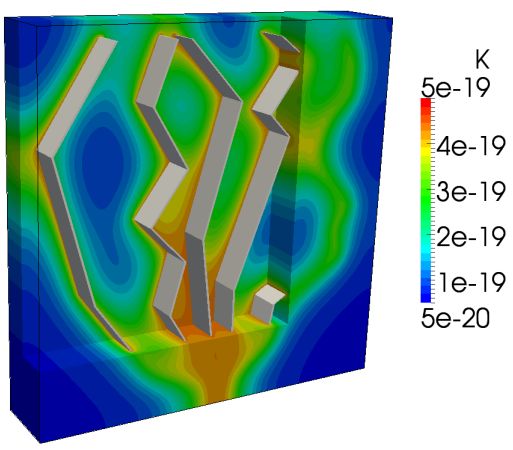

(d) Permeability, Time $=0.36 \mathrm{~h}$

Figure 19: Pressure and permeability distribution vs time, in a complex fracture geometry.

the present investigation are detailed in Table 4 . This model should not be confused with the well known dual porosity model used to represent fractured porous media, as already discussed in Section 2.3. The model presented here does not require any transformation of the physical domain, and treats both regions as completely separate. As a result, a porosity value of $33 \%$ assigned to the fracture in Table 4 represents the porous space within the fracture only, and does not represent the volume of the fracture with respect to the whole fractured domain. In addition, since the fracture has a large aperture compared to the shale matrix which has nanopores, a constant permeability (PM1) was assigned to the fracture, whereas for the matrix both constant 


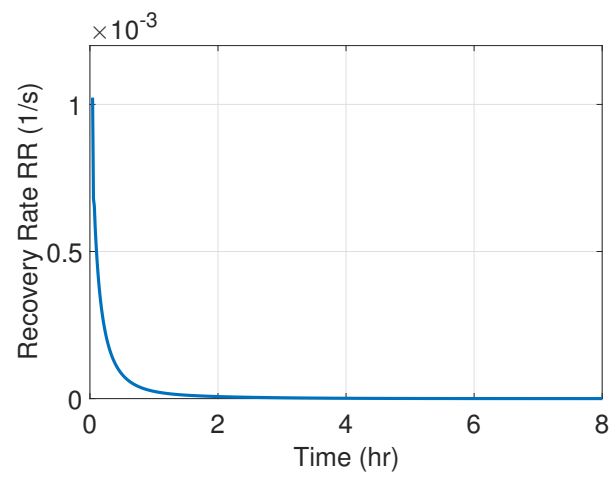

(a) Recovery rate vs. time

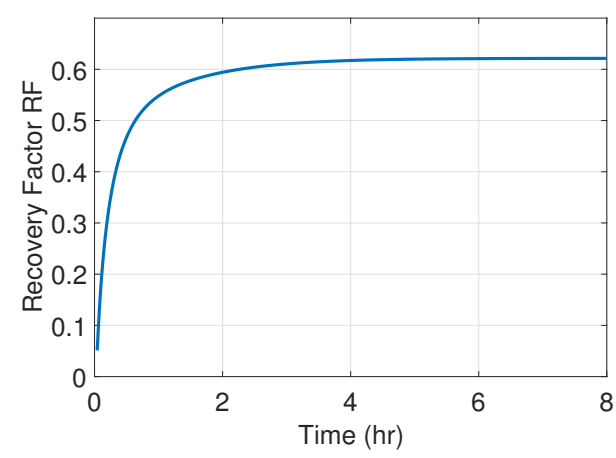

(b) Recovery Factor vs. time

Figure 20: Recovery Rate and Recovery Factor of the complex realistic geometry case.

Table 4: Properties of the matrix and the fracture for the case studied with the dual zone solver.

\begin{tabular}{lll}
\hline \hline Property & Matrix & Fracture \\
\hline Porosity & $9 \%$ & $33 \%$ \\
Permeability & $\mathrm{PM} 1, k=5 \times 10^{-19} \mathrm{~m}^{2}(506 \mathrm{nD})$, or PM3 & $\mathrm{PM} 1, k=5 \times 10^{-15} \mathrm{~m}^{2}(1.013 \mathrm{mD})$ \\
Initial Pore Pressure & $35 \mathrm{MPa}$ & $35 \mathrm{MPa}$ \\
Governing Equation & Eq. 13 & Eq. 13 \\
Common Boundary Condition & Dirichlet BC & Neumann BC \\
Confinement Boundary condition & Neumann zero gradient (wall) & Neumann zero gradient (wall) \\
Outlet & - not applicable- & Dirichlet BC (fixed value) 3MPa \\
\hline \hline
\end{tabular}

permeability (PM1) and apparent permeability function (PM3) were tested, as also summarized in Table 4.

Before looking at the results, it is important to note that the fracture is in direct contact with the low pressure outlet boundary while the matrix is interfaced with the fracture which is at the same initial pressure of the matrix. Furthermore, the fracture permeability is $5 \times 10^{-15} \mathrm{~m}^{2}(1.013 \mathrm{mD})$ compared to $1 \times 10^{-19} \mathrm{~m}^{2}$ (101 nD) for the matrix, at $35 \mathrm{MPa}$. Therefore, the fracture is expected to deplete first and at a faster rate compared to the matrix or, in other words, the pressure in the fracture is expected to balance the outlet pressure of 3.0 MPa long before the matrix. This is shown in Fig. 22, where the pressure field in the case of constant permeability for both the matrix and the fracture are reported. After $5 \mathrm{~s}$ the highest pressure existing in the fracture is around $3.1 \mathrm{MPa}$, whereas a significant volume of the matrix has still a pressure higher than $34.0 \mathrm{MPa}$, although the initial pressure of both the fracture and the matrix was the same (35.0 MPa, see Table 4), indicating that the fracture is almost depleted whereas the matrix 


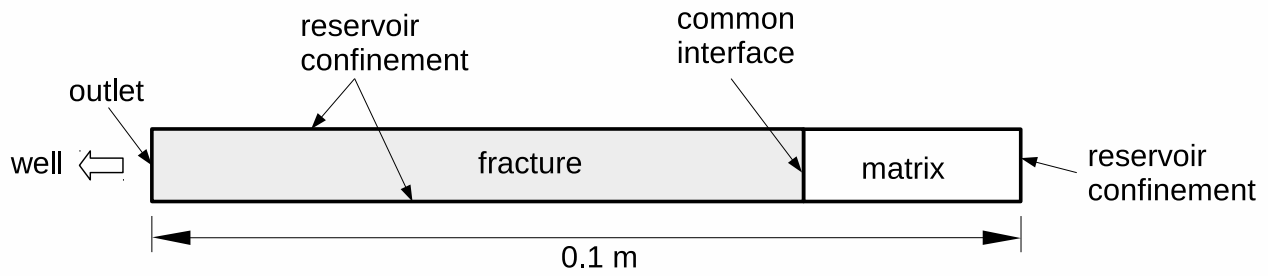

Figure 21: Schematic of the case studied with the dual zone solver.

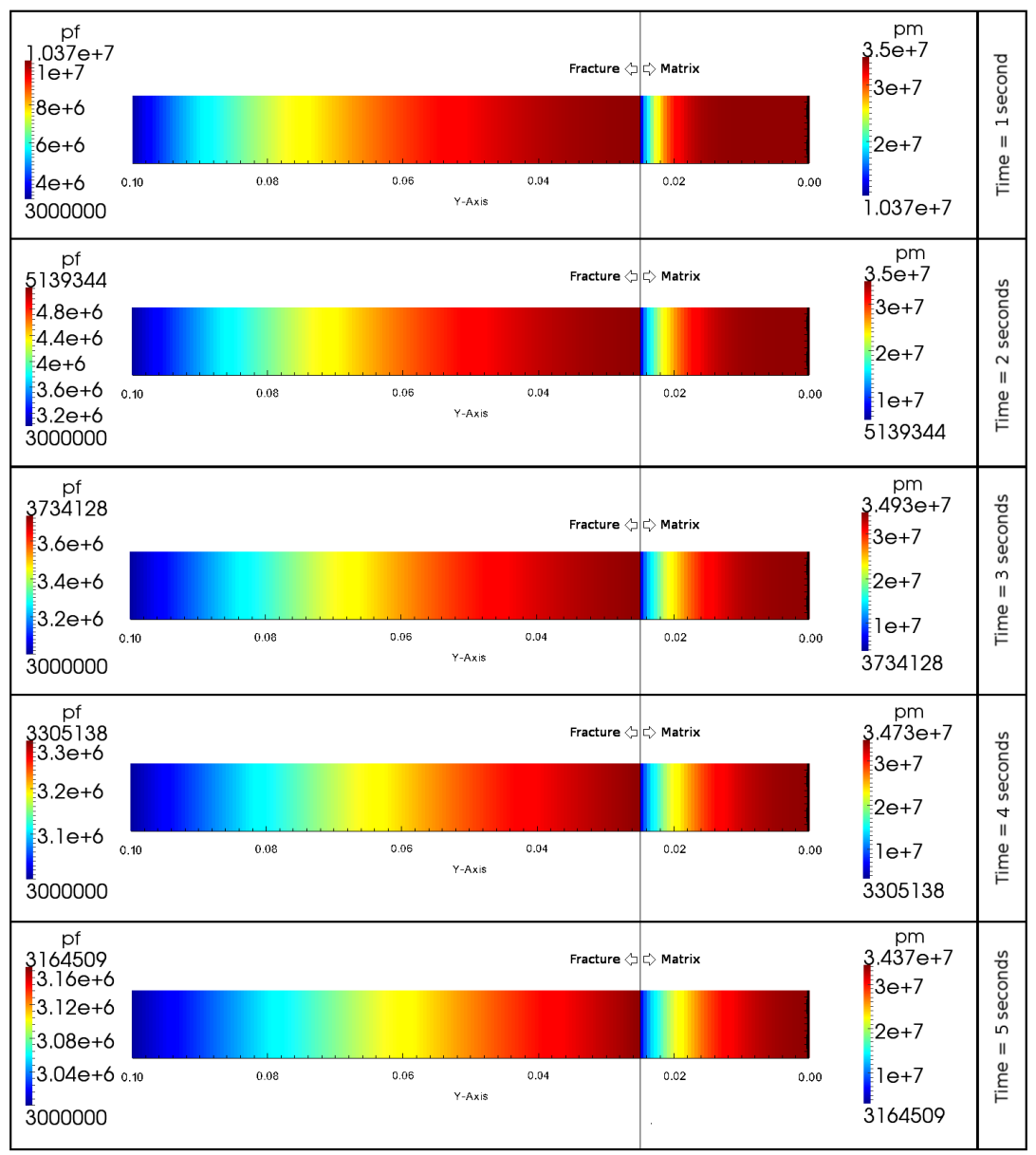

Figure 22: Pressure field in the cross section at different times for the case investigated with the dual zone solver.

still contains the most of the initial shale gas. 


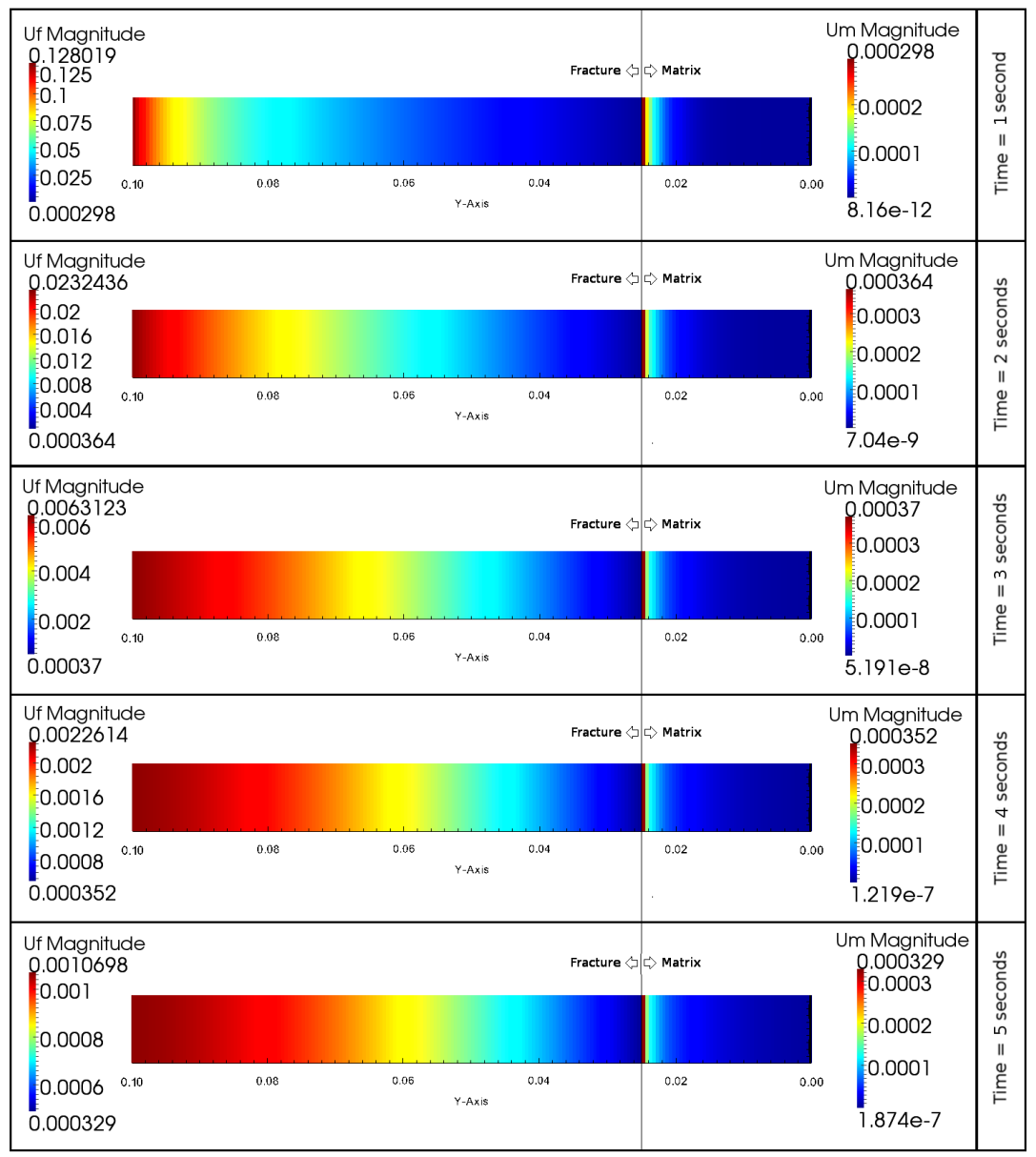

Figure 23: Darcy velocity field in the cross section at different times for the case investigated with the dual solver.

It is also interesting to analyse the Darcy velocity shown in Fig. 23. Consistent with the time evolution of the pressure, the fracture Darcy velocity at the interface with the outlet boundary decreases from $128 \mathrm{~mm} / \mathrm{s}$ at $t=1 \mathrm{~s}$ to about $1 \mathrm{~mm} / \mathrm{s}$ at $t=5 \mathrm{~s}$, whereas values lower than $0.5 \mathrm{~mm} / \mathrm{s}$ are observed at the fracture/matrix interface throughout the transient indicating that the fracture depletes faster than the matrix. Reminding that the Darcy velocity in each region is proportional to the permeability and the pressure gradient (see Eq. 2), the observed behaviour can be related to the time evolution of the pressure as well as the difference in permeability between the 
two zones (the fracture permeability is four orders of magnitude larger than the matrix permeability). At early times the pressure gradient at the fracture/outlet interface is very high and then decreases leading to a decrease of the Darcy velocity, whereas pressure gradients at the fracture/matrix interface are generally low during the whole initial transient causing the lower levels of velocity observed at the common interface. Looking more in detail at the transient behaviour of the Darcy velocity at the common interface, it is interesting to note that, as shown in Fig. 23, the velocity increases from $0.298 \mathrm{~mm} / \mathrm{s}$ at $t=1 \mathrm{~s}$ to $0.37 \mathrm{~mm} / \mathrm{s}$ at $t=3 \mathrm{~s}$ and then decreases back to $0.329 \mathrm{~mm} / \mathrm{s}$ at $t=5 \mathrm{~s}$. Such behaviour can be explained on the basis of the time evolution of the pressure gradient. Since the initial pressure in the two domains, the fracture and the matrix, is the same, at early times the pressure gradient at the interface is very small. However, as the fracture starts to deplete this pressure gradient starts to increase. Owing to the high permeability of the fracture network, the pressure in the fracture drops faster than the pressure in the matrix which has a permeability four orders of magnitude smaller. This fast drop in fracture pressure coupled with a slow drop in matrix pressure, causes the pressure gradient at the boundary in between the two zones to increase rapidly, generating an increase of the velocity. After that, as the pressure in the matrix starts to decrease gradually, the velocity at the interface starts to decrease as well until the complete depletion.

Since the coupling conditions at the common interface impose the same pressure and the same mass flow rate (see Section 2.3 for details), both the pressure and the Darcy velocity at the two sides of the interface should be the same. This is clearly shown in Figs. 22 and 23, thus verifying that the coupling at the boundary is correctly working. This also means that, because of the different values of permeability, the pressure gradient on the matrix side is higher than the one on the fracture side. This has implications for the generation of the mesh. In order to properly resolve the pressure gradient, a higher resolution is generally required on the matrix side of the interface in order to proper resolve

The different behaviour of the Darcy velocity at the outlet boundary and at the fracture/matrix interface has a direct impact on the recovery rate of the two zones, which is directly related to the velocity field. Figures 24(a)-(d) show the recovery factor and recovery rate of the matrix and the fracture, considered as two distinct domains. The recovery rate and the recovery factor of the fracture qualitatively resembles the two regime flow (diffusion dominated) whereas the recovery rate of the matrix (flow rate exiting the 


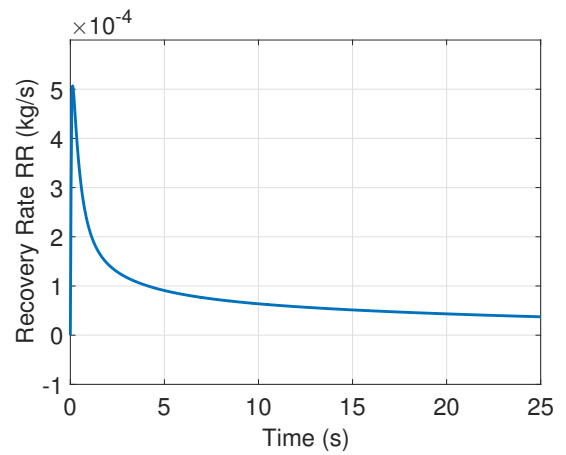

(a) Matrix recovery rate.

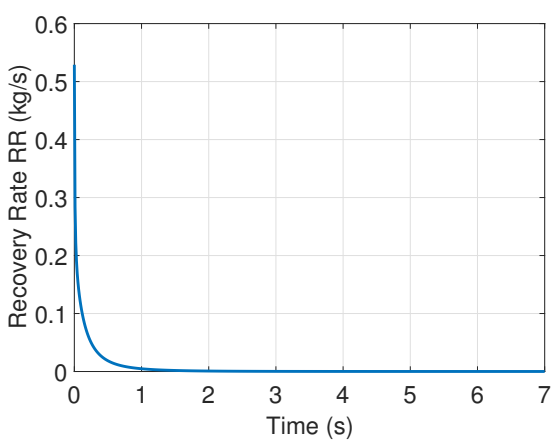

(c) Fracture recovery rate.

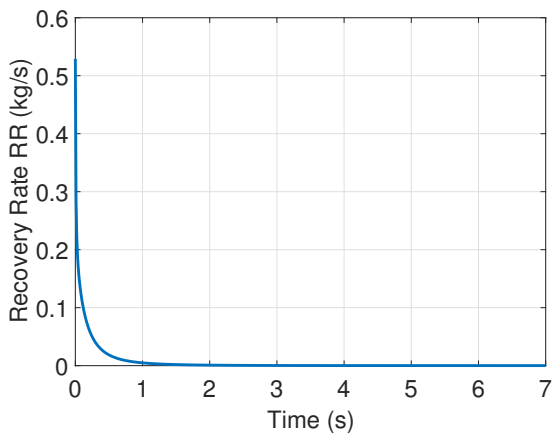

(e) Total recovery rate.

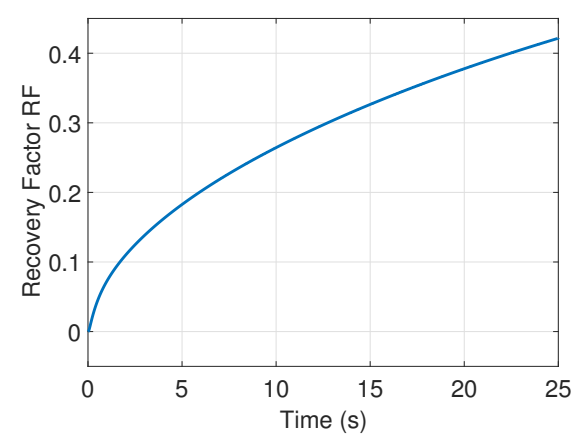

(b) Matrix recovery factor.

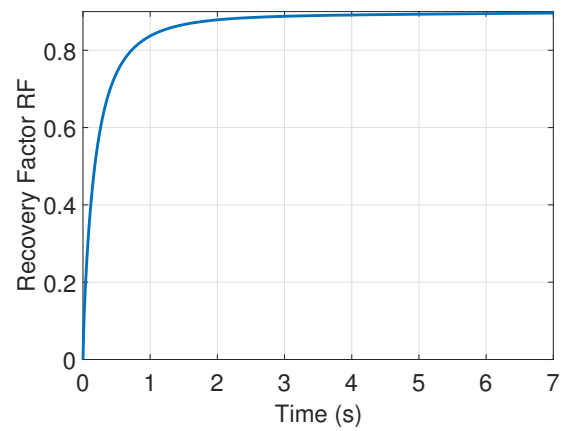

(d) Fracture recovery factor.

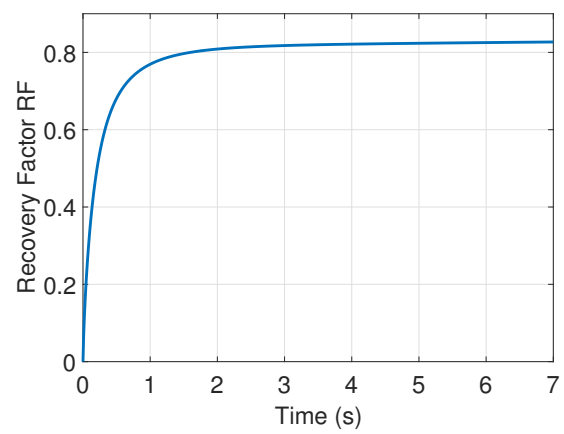

(f) Total recovery factor.

Figure 24: Recovery rate and recovery factor of the matrix, fracture and matrix and fracture combined predicted by the dual zone solver with constant permeability for both the fracture and the matrix.

matrix into the fracture zone, Fig. 24(a)) initially increases followed by a decrease with the typical behaviour observed with the single zone solver 
(the fast drop in recovery rate is unique to shale flow). This is due the previously discussed behaviour of the Darcy velocity at the fracture/matrix interface. Results show a great deal of disparity between the behaviour of flow within the matrix and the fracture, but the overall result of the two domains combined still qualitatively complies with the two regime flow as can be seen in Figs. 24(e)-(f), which represent the recovery factor and recovery rate of the matrix and fracture domains combined as one whole domain.

Results presented so far were obtained with a constant permeability for both the matrix and the fracture. However, different permeability models can be used. For example, a more physically consistent simulation can be performed using an apparent permeability for the matrix, in order to include Knudsen effects. The recovery rate and recovery factor of matrix, fracture and the whole domain (fracture and matrix combined) obtained by assigning the apparent permeability to the matrix are shown in Fig. 25. Results are qualitatively similar to the one obtained for the case with constant permeability (the same applies to the pressure and velocity fields) with some differences due to the change of permeability with pressure (see Fig. 3(b)).

At the very beginning of the simulation, where the pressure is very close to the initial condition, the apparent permeability is lower than $10^{-19} \mathrm{~m}^{2}(101$ $\mathrm{nD})$ and the depletion of the matrix is slower compared to the previous case. The recovery rate should improve during the last stages where because of the smaller pressures the apparent permeability increases.

Results presented in this section provide a fresh attempt at modelling shale flow behaviour, where the coupled behaviour of the fracture and the matrix can be analysed giving more insight into the processes affecting shale gas flow. The flexibility of the code with respect to the physical properties (e.g. permeability model) of the different domains allows us to model the fracture and the matrix with the models and parameters that are more representative of the actual physical behaviour. It should be noted that this dual zone approach can be very expensive (in terms of computational cost) for cases characterized by a very large and intricate fracture network. However, the detailed results that can be obtained with this approach can be exploited to assess and possibly calibrate low-order models for shale gas flow. 


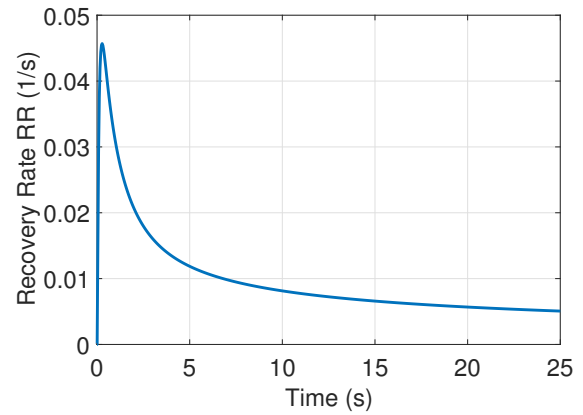

(a) Matrix recovery rate.

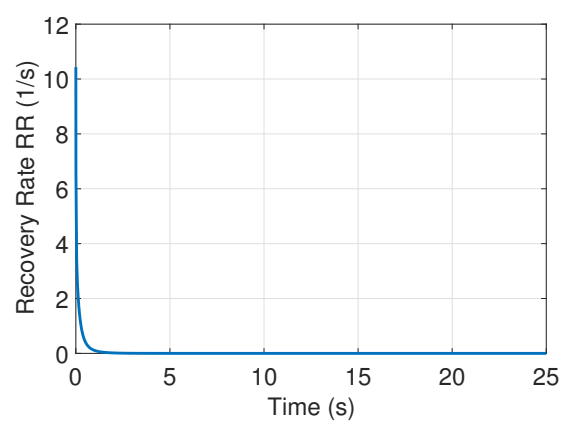

(c) Fracture recovery rate.

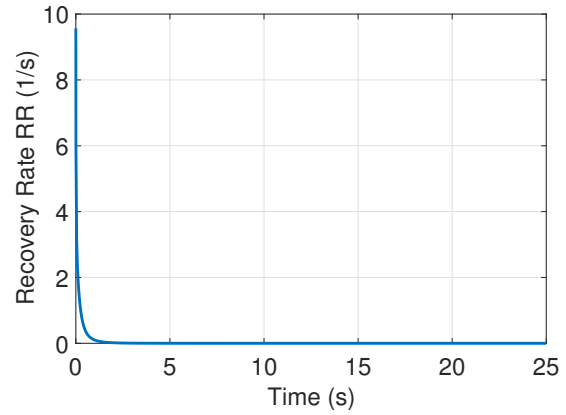

(e) Total recovery rate.

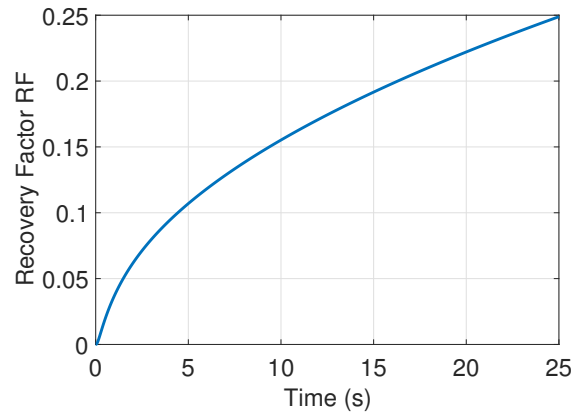

(b) Matrix recovery factor.

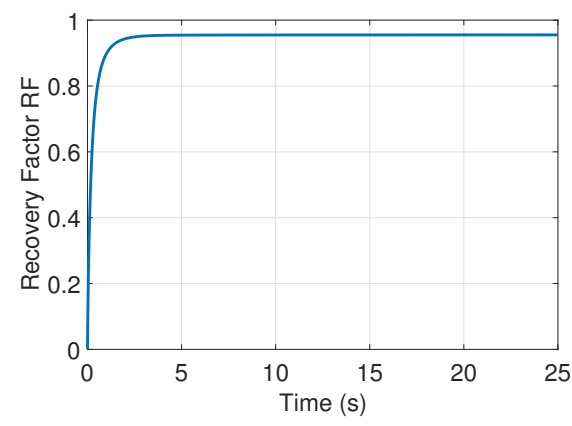

(d) Fracture recovery factor.

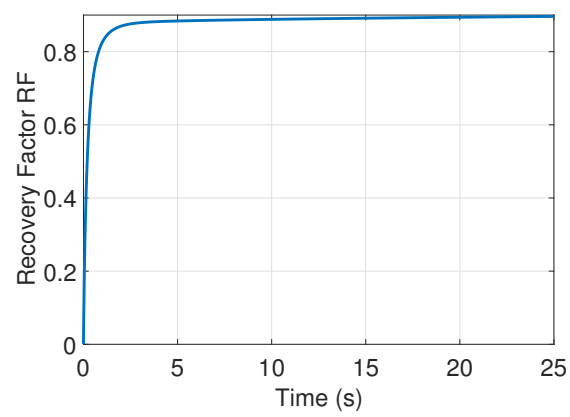

(f) Total recovery factor.

Figure 25: Recovery rate and recovery factor of the matrix, fracture and matrix and fracture combined predicted by the dual zone solver with apparent permeability for the matrix and constant permeability for the fracture.

\section{Conclusion}

This study presented a three dimensional CFD solver to simulate shale gas flow in porous media utilizing the OpenFOAM framework. A tailored 
governing equation that does not require the use of pseudo pressures, because the numerical solver implements the gas properties as functions of pressure, was utilized. The proposed approach allows using different relations for the properties on the basis of the case under investigation or the choice of the user. First, the solver was validated against the findings of Patzek et al. (2013) and Freeman et al. (2013). It was found that the recovery rate and the recovery factor match a two regime flow. The rate of increase in recovery factor, $\kappa$, depends on the fracture geometry, permeability, and initial conditions. Then, four permeability models were implemented to account for stress effects and Knudsen and slip flow. The results confirmed the dominance of the diffusion aspect on shale flow, as the results were comparable and the trends were similar between the governing equation and a diffusion equation. In addition, it was noted that the fracture network geometry imposes the recovery rate. The flow was indistinguishable, even after fracture interference, for four reservoirs with the same fracture network but with different reservoir dimensions. Finally, an extension was developed based on the single solver, named the dual solver, drawing inspiration from the dual porosity/dual permeability models as well as previous heat transfer models. The physical geometry is preserved and directly solved, without any transformation. The dual solver treats the matrix and the fracture network as two separate zones, each with its own governing equations and parameters. The recovery rate of the whole domain qualitatively adhered to the diffusion dominated two regime flow. Different permeability models were also implemented.

\section{Appendix A. Additional Results}

As noted in Sections 2.4.1 and 2.4.3, additional results were produced for Case 2, using the viscosity and permeability relations given by Eqs. B.1 and B.2 in order to assess the effect of using different viscosity and compressibility factor relations proposed in literature. This further points out the flexibility of the proposed approach, which allows implementing any gas property relation, as previously discussed in Section 2.4.

Fig. A.26 shows that, for the investigated case, recovery factor and recovery rate still adhere to the two regime flow described in Section 3.1.1. When comparing Fig. A.26 with Fig. 9(a,c) and Fig. 11(a,c), the variation in results between using Eqs. 17, 20 and Eqs. B.1, B.2 is minor, and the same slopes are used to match the results. 


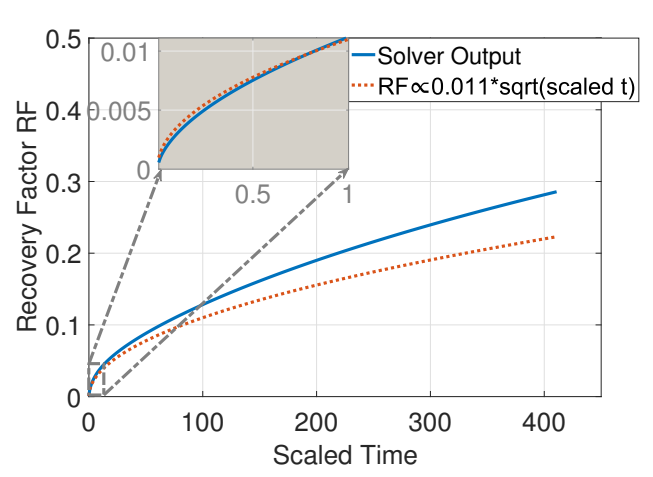

(a) Recovery Factor, PM1

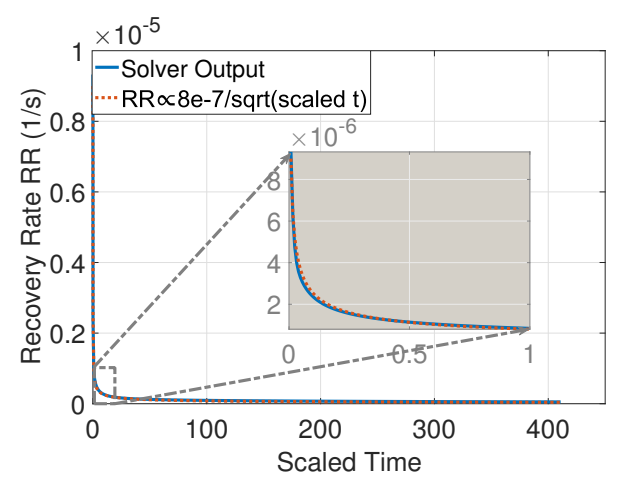

(c) Recovery Rate, PM1.

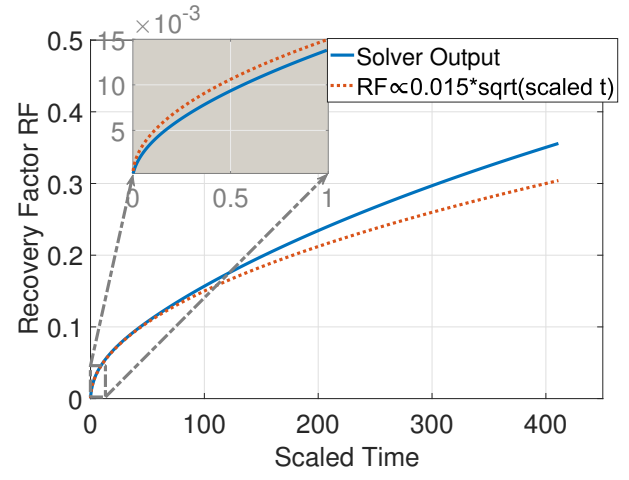

(b) Recovery Factor, PM3

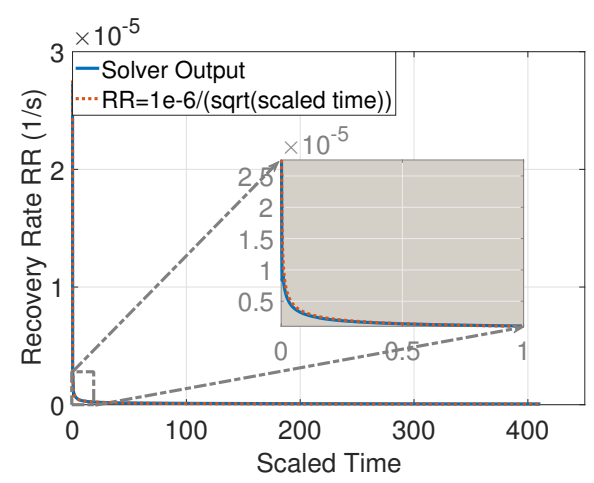

(d) Recovery Rate, PM3.

Figure A.26: Recovery rate and recovery factor versus scaled time for Case 2 predicted using the equation of state and the viscosity relationships from Jarrahian et al. (2015); Jarrahian and Heidaryan (2014) (Eq. B.1 and Eq. B.2, with curve matching.)

Fig. A.27 shows the geometry of the case referred to in Section 3.3, when the change in width is tested. Fig. A.28 indicates that the results are the similar to that of varying length. The same conclusion can be made; the flow behaviour is determined by the fracture network. 


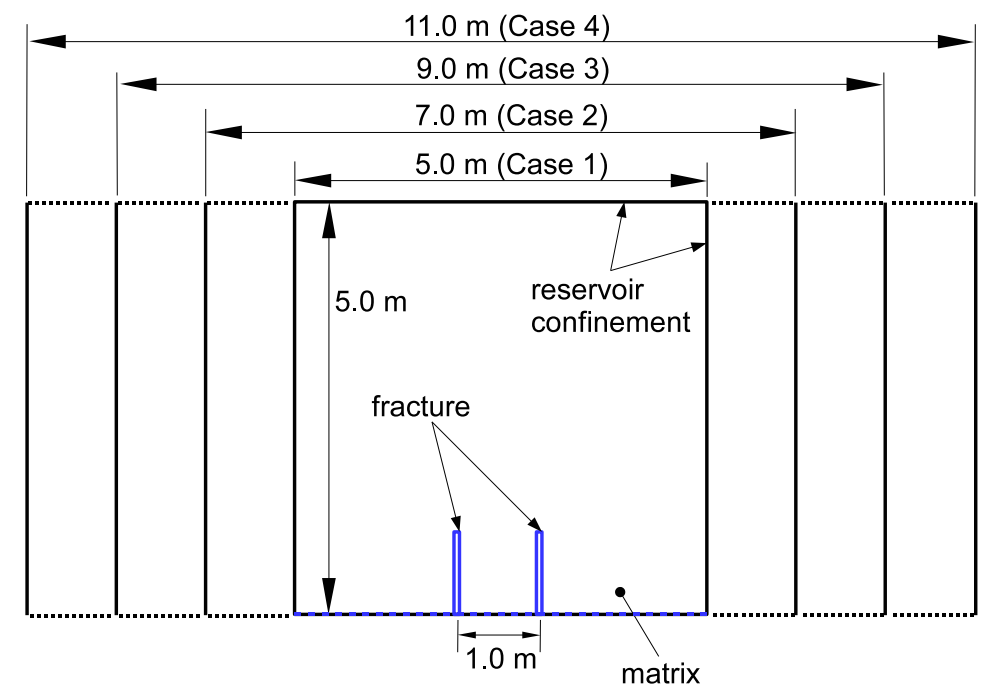

Figure A.27: Schematic of the extra cases considered for the sensitivity analysis to the reservoir dimension.
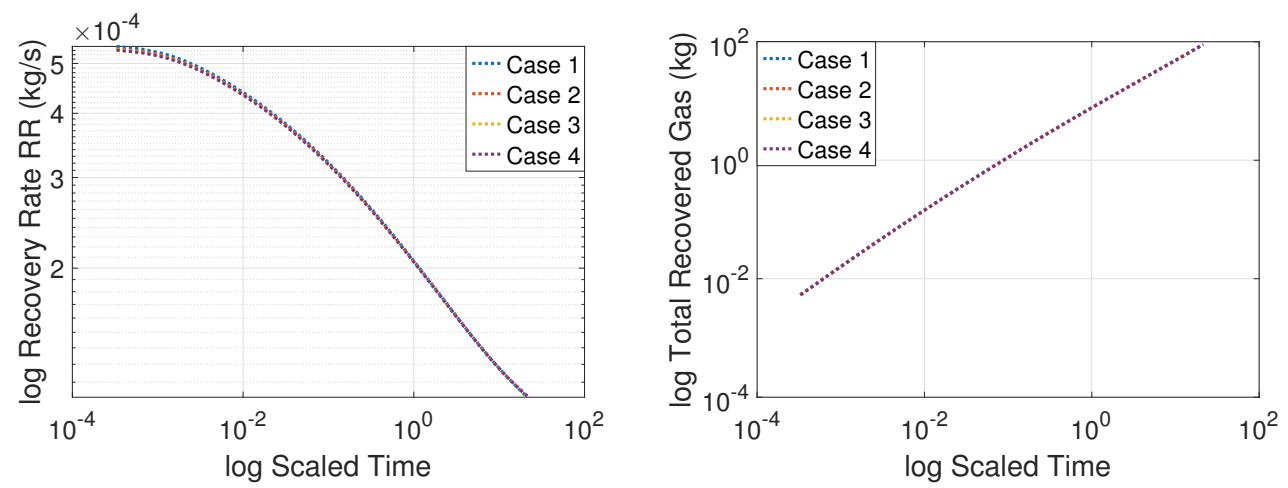

(a) Logarithmic plot of recovery rate vs. (b) Logarithmic plot of recovery vs. scaled scaled time time

Figure A.28: Sensitivity analysis to the dimension of the reservoir.

\section{Appendix B. Additional Equations}

Appendix $\mathrm{B}$ includes the equations referred to, but not included in the body of this paper.

$$
\begin{gathered}
z=\frac{P V}{R T}=1+\frac{1}{V}\left(b-\frac{a \beta}{R T}\right)+\frac{1}{V^{2}} b\left(b+\frac{a \beta}{R T}\right) \\
\beta=\beta_{1}+\beta_{2} L n P_{p r}+\frac{\beta_{3}}{T_{p r}}+4 \beta_{4} L n^{2} P_{p r}+\frac{\beta_{5}}{T_{p r}^{2}}+\frac{\beta_{6} L n P_{p r}}{T_{p r}} \\
a=0.49694 \frac{\left(R T_{p C}\right)^{2}}{P_{p C}} \\
b=0.09012 \frac{R T_{p C}}{P_{p C}}
\end{gathered}
$$




$$
\begin{gathered}
\mu_{g}=1+\left(A_{1}+\frac{A_{2}}{T_{p r}}+\frac{A_{3}}{T_{p r}^{3}}\right) \rho_{R}+\left(A_{4}+\frac{A_{5}}{T_{p r}}\right) \rho_{R}^{2}+\left(\frac{A_{5} A_{6}}{T_{p r}}\right) \rho_{R}^{5}+\left(\frac{A_{7}}{T_{p r}^{3}}\right) \rho_{R}^{2}\left(1+{ }_{8} \rho_{R}^{2}\right) \operatorname{Exp}\left(-A_{8} \rho_{R}^{2}\right) \\
\mu_{a t m}=\frac{C_{1} T_{p r}^{C_{2}}+C_{3} \operatorname{Exp}\left(C_{4} T_{p r}\right)+C_{5} \operatorname{Exp}\left(C_{6} T_{p r}\right)+C_{7}}{\zeta}+\Delta \mu\left(H_{2}\right) \\
\rho_{R}=\frac{P_{p r}}{\left(B_{1}+B_{2} \gamma_{g}\right) Z T_{p r}} \\
\zeta={\frac{T_{p C}}{M_{w}^{3} P_{p C}^{4}}}^{\frac{1}{6}}
\end{gathered}
$$

where $\beta_{1}, \beta_{2}, \beta_{3}, \beta_{4}, \beta_{5}, \beta_{6}, T_{p r}, P_{p r}, P_{p C}, T_{p C}$ are provided in Jarrahian and Heidaryan (2014).

where $A_{1}, A_{2}, A_{3}, A_{4}, A_{5}, A_{6}, A_{7}, A_{8}, B_{1}, B_{2}, C_{1}, C_{2}, C_{3}, C_{4}, C_{5}, C_{6}, C_{7}$, $T_{p r}, P_{p r}, P_{p C}, T_{p C}$, and $\Delta \mu\left(H_{2}\right)$ are provided in Jarrahian et al. (2015).

\section{Appendix C. Nomenclature}

\section{Symbols}

A Surface Area, $\mathrm{m}^{2}$

$C_{g} \quad$ Gas Compressibility, $1 / \mathrm{Pa}$

$C_{r} \quad$ Reduced Gas Compressibility

$d \quad$ Fracture Spacing, $m$

$h \quad$ Elevation, $m$

$k \quad$ Absolute Permeability, $\mathrm{m}^{2}$

$m \quad$ Recovered gas mass, $\mathrm{kg}$

$M \quad$ Initial gas mass, $\mathrm{kg}$

$P \quad$ Pressure, $\mathrm{Pa}$

$P_{c r} \quad$ Critical Pressure, $\mathrm{Pa}$

$P M \quad$ Permeability Model

$R F \quad$ Recovery Factor

$t \quad$ Time, s

$\tilde{t} \quad$ Scaled Time

$T_{c r} \quad$ Critical Temperature, $\mathrm{K}$ 


$\begin{array}{ll}u & \text { Darcy Velocity, m/s } \\ M_{w} & \text { Molar Mass, kg/kmol } \\ r & \text { Pore Radius, m } \\ R & \text { Universal Gas Constant, } 8.314 \mathrm{~J} /(\text { mol.K }) \\ z & \text { Compressibility Factor } \\ \alpha & \text { Tangential Momentum Accommodation Coefficient } \\ \phi & \text { Porosity } \\ \mu & \text { Viscosity, Pa.s } \\ \rho & \text { Density, kg/m }{ }^{3} \\ \tau & \text { Characteristic Interference Time, s } \\ & \\ \text { Subscript } & \\ & \\ a & \text { Apparent } \\ \text { avg } & \text { Average } \\ f & \text { Fracture } \\ m & \text { Matrix } \\ n & \text { Normal component }\end{array}$

\section{References}

Blasingame, T. A. and Poe, B. D. (1993). Semianalytic solutions for a well with a single finite-conductivity vertical fracture. Society of Petroleum Engineers.

Bruner, K. R., , and Smosna, R. (2011). A comparative study of the mississippian barnett shale, fort worth basin, and devonian marcellus shale, appalachian basin. National Energy Technology Laboratory. U.S. Department of Energy. (from Web. 18 Aug. 2015).

Brush, D. J. and Thomson, N. R. (2003). Fluid flow in synthetic rough-walled fractures: Navier-stokes, stokes, and local cubic law simulations. Water Resources Research, 39(4).

Chen, Z., Huan, G., and Ma, Y. (2006). Computational Methods for Multiphase Flows in Porous Media (Computational Science and Engineering 2). Society for Industrial and Applied Mathematics, Philadelphia, PA, USA.

Cipolla, C. L., Lolon, E., Erdle, J., and Tathed, V. S. (2009). Modeling well performance in shale-gas reservoirs. In SPE/EAGE Reservoir Char- 
acterization and Simulation Conference, 19-21 October, Abu Dhabi, UAE, number SPE-125532-MS. Society of Petroleum Engineers.

Craven, B. and Campbell, R. (2011). Multi-region conjugate heat/mass transfer mrconjugateheatfoam: A dirichletneumann partitioned multi-region conjugate heat transfer solver. http://www.personal.psu.edu/dab143/OFW6/training.htm.

ERCB (1979). Gas Well Testing, Theory and Practice. Energy Resources Conservation Board.Directive 034, Alberta Energy Regulator, Calgary, Canada.

Fan, X., Li, G., Shah, S. N., Tian, S., Sheng, M., and Geng, L. (2015). Analysis of a fully coupled gas flow and deformation process in fractured shale gas reservoirs. Journal of Natural Gas Science and Engineering.

Fan, Y., Durlofsky, L., and Tchelepi, H. A. (2010). Numerical simulation of the in-situ upgrading of oil shale. SPE Journal, (SPE-118958-PA).

Freeman, C., Moridis, G., and Blasingame, T. (2011). A numerical study of microscale flow behavior in tight gas and shale gas reservoir systems. Transport in Porous Media, 90(1):253-268.

Freeman, C., Moridis, G., Ilk, D., and Blasingame, T. (2013). A numerical study of performance for tight gas and shale gas reservoir systems. Journal of Petroleum Science and Engineering, 108:22 - 39.

Friend, D. G., Ely, J. F., and Ingham., H. (1989). Thermophysical properties of methane. Journal of Physical and Chemical Reference Data, 18.2:583.

Gonzalez, M., Eakin, B., and Lee, A. (1970). Viscosity of natural gases. Monograph on API Research Project, Institute of Gas Technology (Chicago), (65).

Gringarten, A., Ramey, H., and Raghavan, R. (1974). Unsteady-state pressure distributions created by a well with a single infinite-conductivity vertical fracture. Society of Petroleum Engineers Journal, 14(4).

Gruber, J. (2014). Fluid mechanics of shale-gas recovery. Master's thesis, University of Cambridge. 
Hill, D. and Nelson, C. (2000). Gas productive fractured shales: An overview and update. Gas TIPS, 6(3):4-13.

Houze, O., Tauzin, E., Artus, V., and Larson, L. (2010). The analysis of dynamic data in shale gas reservoirs. Kappa Engineering, Houston, Texas, USA.

Jarrahian, A., Aghel, B., and Heidaryan, E. (2015). On the viscosity of natural gas. Fuel, 150:609-618.

Jarrahian, A. and Heidaryan, E. (2014). A new cubic equation of state for sweet and sour natural gases even when composition is unknown. Fuel, 134:333-342.

Javadpour, F. (2009). Nanopores and apparent permeability of gas flow in mudrocks (shales and siltstone). Journal of Canadian Petroleum Technology, 48(8).

Jayakumar, R., Sahai, V., and Boulis, A. (2011). A better understanding of finite element simulation for shale gas reservoirs through a series of different case histories. In SPE Middle East Unconventional Gas Conference and Exhibition, 31 January-2 February, Muscat, Oman, number SPE-142464MS. Society of Petroleum Engineers.

Klinkenberg, L. J. (1941). The permeability of porous media to liquids and gases. American Petroleum Institute.

Kwon, O., Kronenberg, A., Gangi, A., and Johnson, B. (2001). Permeability of wilcox shale and its effective pressure law. J. Geophys. Res., 106(B9).

Ling, K. (2010). Gas Viscosity at High Pressure and High Temperature. PhD thesis, Texas A\&M University.

Ma, J., Sanchez, J. P., Wu, K., Couples, G. D., and Jiang, Z. (2014). A pore network model for simulating non-ideal gas flow in micro- and nano-porous materials. Fuel, 116:498 - 508.

Mahmoud, M. (2013). Development of a new correlation of gas compressibility factor (z-factor) for high pressure gas reservoirs. In SPE North Africa Technical Conference and Exhibition, 15 April-17 April, Cairo, Egypt, number SPE-164587. Society of Petroleum Engineers. 
Milici, R. C. and Swezey, C. S. (2006). Assessment of appalachian basin oil and gas resources: Devonian shale middle and upper paleozoic total petroleum system. Reston, Virginia: U.S. Department of the Interior, U.S. Geological Survey. Web. Open-File Report Series.

Miller, M. A., Jenkins, C. D., and Rai, R. R. (2010). Applying innovative production modeling techniques to quantify fracture characteristics, reservoir properties, and well performance in shale gas reservoirs. In SPE Eastern Regional Meeting, 13-15 October, Morgantown, West Virginia, USA, number SPE-139097-MS. Society of Petroleum Engineers.

Mohaghegh, S. (2013). Reservoir modeling of shale formations. Journal of Natural Gas Science and Engineering, 12:22 - 33.

Moridis, G. and Freeman, C. (2014). The realgas and realgash2o options of the tough+ code for the simulation of coupled fluid and heat flow in tight/shale gas systems. Computers $\& 3$ Geosciences, 65:56 - 71 .

Moridis, G. J., Blasingame, T. A., and Freeman, C. M. (2010). Analysis of mechanisms of flow in fractured tight-gas and shale-gas reservoirs. In SPE Latin American and Caribbean Petroleum Engineering Conference, 1-3 December, Lima, Peru, number SPE-139250-MS. Society of Petroleum Engineers.

Patzek, T. W., Male, F., and Marder, M. (2013). Gas production in the Barnett Shale obeys a simple scaling theory. Proceedings of the National Academy of Sciences, 110(49):19731-19736.

Peng, D.-Y. and Robinson, D. B. (1975). A new two-constant equation of state. Ind. Eng. Chem., Fundam., 15:59-64.

Shabro, V., Torres-Verdin, C., and Sepehrnoori, K. (2012). Forecasting Gas Production In Organic Shale With The Combined Numerical Simulation Of Gas Diffusion In Kerogen, Langmuir Desorption From Kerogen Surfaces, And Advection In Nanopores. In SPE Annual Technical Conference And Exhibition.

Soave, G. (1972). Equilibrium constants from a modified redlich-kwong equation of state. Chemical Engineering Science, 27:1197-1203. 
Soeder, D. J. (1988). Porosity and permeability of eastern devonian gas shale. SPE Formation Evaluation, 3(1).

Taylor, T. (2013). Lithostratigraphic and petrophysical analysis of the middle devonian marcellus shale at the mamont prospect, westmoreland county, pennsylvania. Master's thesis, Clemson University.

Wang, Q., Chen, X., Jha, A. N., and Rogers, H. (2014). Natural gas from shale formation. the evolution, evidences and challenges of shale gas revolution in united states. Renewable and Sustainable Energy Reviews, 30:1 -28 .

Weller, H. G., Tabor, G., Jasak, H., and Fureby, C. (1998). A tensorial approach to computational continuum mechanics using object-oriented techniques. Computers in Physics, 12(6).

Wu, K., Chen, Z., Wang, H., Yang, S., Li, X., and Shi, J. (2015). A model for real gas transfer in nanopores of shale gas reservoirs. In $E U$ ROPEC, 1 June-4 June, Madrid, Spain, number SPE-174293-MS. Society of Petroleum Engineers.

Youtsos, M., Mastorakos, E., and Cant, R. (2013). Numerical simulation of thermal and reaction fronts for oil shale upgrading. Chemical Engineering Science, 94:200 - 213.

Yu, W., Luo, Z., Javadpour, F., Varavei, A., and Sepehrnoori, K. (2014). Sensitivity analysis of hydraulic fracture geometry in shale gas reservoirs. Journal of Petroleum Science and Engineering, 113:1 - 7.

Zimmerman, R. and Bodvarsson, G. S. (1996). Hydraulic conductivity of rock fractures. Transport in Porous Media, 23(1):1-30. 